\title{
Evaluation of Reaction Rate Theory and Monte Carlo Methods for Application to Radiation-Induced Microstructural Characterization
}

\author{
R. E. Stoller ${ }^{1}$, S. I. Golubov ${ }^{2}$, C. Domain ${ }^{3}$, and C. S. Becquart ${ }^{4}$ \\ ${ }^{1}$ Materials Science and Technology Division, Oak Ridge National Laboratory, P. O. Box 2008, \\ Oak Ridge, TN 37831-6158 USA \\ ${ }^{2}$ Center for Materials Processing, University of Tennessee, East Stadium Hall, Knoxville, TN \\ 37996-0750, USA \\ ${ }^{3}$ EDF-R\&D Département MMC, Les Renardières, F-77818 Moret sur Loing Cédex, France \\ ${ }^{4}$ Laboratoire de Métalurgie Physique et Génie des Matériaux, UMR 8517, Université Lille-1, F- \\ 59655 Villeneuve d'Ascq Cédex, France
}

\begin{abstract}
The multiscale modeling scheme encompasses models from the atomistic to the continuum scale. Phenomena at the mesoscale are typically simulated using reaction rate theory, Monte Carlo, or phase field models. These mesoscale models are appropriate for application to problems that involve intermediate length scales, and timescales from those characteristic of diffusion to longterm microstructural evolution ( $\sim \mu$ s to years). Although the rate theory and Monte Carlo models can be used simulate the same phenomena, some of the details are handled quite differently in the two approaches. Models employing the rate theory have been extensively used to describe radiation-induced phenomena such as void swelling and irradiation creep. The primary approximations in such models are time- and spatial averaging of the radiation damage source term, and spatial averaging of the microstructure into an effective medium. Kinetic Monte Carlo models can account for these spatial and temporal correlations; their primary limitation is the computational burden which is related to the size of the simulation cell. A direct comparison of $\mathrm{RT}$ and object kinetic MC simulations has been made in the domain of point defect cluster dynamics modeling, which is relevant to the evolution (both nucleation and growth) of radiationinduced defect structures. The primary limitations of the OKMC model are related to computational issues. Even with modern computers, the maximum simulation cell size and the maximum dose (typically much less than $1 \mathrm{dpa}$ ) that can be simulated are limited. In contrast, even very detailed RT models can simulate microstructural evolution for doses up 100 dpa or greater in clock times that are relatively short. Within the context of the effective medium, essentially any defect density can be simulated. Overall, the agreement between the two methods is best for irradiation conditions which produce a high density of defects (lower temperature and higher displacement rate), and for materials that have a relatively high density of fixed sinks such as dislocations.
\end{abstract}




\section{Introduction}

As discussed in a previous report [1], an adequate quantitative understanding of the changes in the physical and mechanical properties of materials that occur during thermal aging or under irradiation requires a model capable of describing the formation and growth of point defect and solute clusters through all stages of cluster evolution, from their nucleation to growth and coarsening. That report provided a general description of two alternate methods, reaction rate theory [2] and object kinetic Monte Carlo, that can be used to describe cluster evolution and some preliminary comparisons of the results obtained from specific implementations of the two methods. A substantial fraction of the background material from that preliminary report is repeated here in order to provide a self-contained description of both the computational methods and an extensive comparison of the predictions the models provide.

If a random spatial distribution of defect clusters is assumed, their evolution can be described in terms of a defect size distribution function (SDF) in the framework of the so-called mean field approximation. Evolution of this SDF can be accounted in the context of a reaction rate theory (RT) model though the use of a Master Equation (ME) that describes both growth and dissolution of the clusters due to reactions with mobile defects (or solutes), thermal emission of these same species, and cluster coalescence if the clusters are mobile. The relevant physical processes require accounting for clusters containing a very large number of point defects or atoms $\left(>10^{6}\right)$, particularly for high irradiation doses or long ageing times. An explicit discretization of the ME leads to a system of coupled differential equations in which time is an explicit variable. The number of equations is the same as the number of point defects (an/or solutes) in the largest possible cluster. Numerical integration of such a system is feasible on modern computers, but such calculations are overly time consuming. Most practicable solutions to the ME by numerical methods employ some grouping procedure to significantly reduce the number of equations [3-8].

The object kinetic Monte Carlo technique (OKMC) provides another method for calculating the evolution of the cluster SDF. This method has become practicable during last decade because of progress in computer technology. In the framework of OKMC, each point defect or defect cluster is treated as an object located in a specific position in a simulation cell of a given volume. Irradiation is simulated by introducing new point defects or defect clusters at 
discrete times and in specific locations in the box, and evolution occurs as the various objects migrate and participate in a series of predefined reactions with other objects. Each object type has associated properties, such as size, reaction radius, and, if mobile, jump frequency and activation energy for migration. The reaction probabilities for various physical transition mechanisms, such as migration jump rates and the emission rate of point defects from larger defects or traps, are calculated based on Arrhenius frequencies for thermally activated events. The Monte Carlo algorithm [9] is used at each step to select the event that is going to take place based on the corresponding probabilities. After a given event is chosen, the time is increased according to a algorithm that depends on the jump frequencies for all the possible events in the system.

One advantage of the RT approach is there are essentially no limits to the density or size of the clusters when calculating their evolution, providing the opportunity to compare with a broad range of experimental observations. However, the spatial and temporal correlations in defect production are not accounted for, which may in some cases lead to a loss of specific information. OKMC models can account for these spatial and temporal correlations; their primary limitation is related to the size of the simulation cell. In order to maintain a reasonable simulation time, the practicable box size is typically a cube with an edge length on the order of $100 \mathrm{~nm}$. This limits the total cluster number density which may be obtained in this method. For example, if there is only one cluster in a $100 \mathrm{~nm} \times 100 \mathrm{~nm} \times 100 \mathrm{~nm}$ simulation cell, the corresponding cluster number density is $10^{21} \mathrm{~m}^{-3}$. This is an intermediate to high defect density for many relevant irradiated materials, and no lower cluster density can be treated by OKMC in a box of this size. Even for the relatively high defect density of $10^{22} \mathrm{~m}^{-3}$, the number of clusters in such a simulation cell would only be 10 , which may not be enough to provide a good statistical simulation of defect cluster evolution. Another problem with statistics may arise when simulating clusters which are not stable in a certain size range, i.e. when cluster nucleation requires reaching a certain critical size at which they become stable enough to be able grow continuously. Such nucleation is possible due to fluctuations in the cluster growth and resolution processes and will be properly calculated by OKMC only if the density is sufficiently high to maintain such fluctuations.

Thus, both the RT and OKMC have advantages and limitations based on the underlying nature of the techniques; the major features of these models are briefly summarized in Table 1. 
Since the two techniques simply represent different mathematical methods for solving a given problem, they should provide similar results for well designed sample problems. A systematic application of the two methods will determine if they produce consistent results, or whether there are problems for which one method is preferable to the other. This is the main objective of the present study. Since the RT approach has been well established by broad use during last few decades, the approach taken here was to use RT calculations as a reference point to evaluate the predictions of the OKMC method for several different types of simulations. The RT and OKMC models used for this comparison are presented in the next section. The results of the calculations and a discussion of the conclusions are given in Sections 3 and 4, respectively.

Table 1: Comparison of reaction rate theory and object kinetic Monte Carlo methods

\begin{tabular}{|c|c|c|}
\hline $\begin{array}{c}\text { Feature or Treatment } \\
\text { of Variable }\end{array}$ & Reaction Rate Theory & Object Kinetic Monte Carlo \\
\hline solution method & deterministic & stochastic \\
\hline time & explicit variable & $\begin{array}{l}\text { inferred from possible } \\
\text { processes and reaction rates }\end{array}$ \\
\hline space & $\begin{array}{l}\text { homogeneous effective } \\
\text { medium }{ }^{1}\end{array}$ & full spatial dependence \\
\hline defect production & time and space-averaged $^{2}$ & discrete in time and space \\
\hline sink strength & explicit analytical expression & $\begin{array}{l}\text { inferred from fate of point } \\
\text { defects }\end{array}$ \\
\hline defect or sink density & essentially unlimited & $\begin{array}{l}\text { computationally limited by } \\
\text { simulation cell size, i.e.: } \\
\mathrm{N}_{\min } \geq 1 /(\text { box volume })\end{array}$ \\
\hline \multicolumn{3}{|c|}{$\begin{array}{l}1,2 \text { partial corrections to the typical RT approximations are possible, including multi-region } \\
\text { RT models to account for spatial dependence in a limited way and the use of a so-called } \\
\text { cascade diffusion model [10] to treat the time dependence of primary damage formation }\end{array}$} \\
\hline
\end{tabular}

\section{Description of Models}

The comparison of the two methods is more straight forward if restricted to a particular case. This investigation is focused on the evolution of the size distribution function of vacancy 
and self-interstitial atoms (SIAs) clusters in a pure metal under irradiation with a constant network dislocation density as the only fixed sink. In the following discussion, the term void is used in a generic way to refer to a vacancy cluster of any size. The formulated model makes use of the following assumptions:

1. The primary damage is produced either: (a) in the form of Frenkel pair, i.e. only single vacancies and SIAs; or (b) Frenkel pair plus small clusters of either vacancies or SIAs typical of those generated by displacement cascades;

2. The point defects diffuse by three-dimensional random walk;

3. The nucleation of vacancy and SIA clusters proceeds via a homogeneous mechanism, that is due to monodefect + monodefect $=$ di-defect in the case of 1(a), or by both homogeneous and in-cascade clustering mechanisms at the same time for 1(b);

4. Vacancy clusters are treated as spherical voids that are neutral sinks for point defects; thermal emission of vacancies is determined by a size-dependent binding energy;

5. SIA clusters are treated as either planar loops or three dimensional spherical clusters, with a preference (bias) for absorbing SIAs relative to vacancies, and are stable against thermal SIA emission;

6. Edge dislocations are a fixed matrix sink with a preference (bias) for SIAs that is the same as the SIA clusters; and

7. The vacancy and SIA clusters are immobile.

These assumptions are only used for simplification and do not lead to any restrictions in comparing the two methods.

\subsection{Rate theory model}

\subsubsection{Form of master equation}

Based on the assumptions listed above, the SDF of voids, $f_{v}(x, t)$, and SIA clusters, $f_{i}(x, t)$, can be described by the following pair of master equations, in which $\mathrm{x}$ is the number of point defects in the cluster $[4,11]$ :

$$
\begin{aligned}
& \frac{\partial f_{v}(x, t)}{\partial t}=K_{v}(x)+J_{v}(x-1, t)-J_{v}(x, t), \\
& \frac{\partial f_{i}(x, t)}{\partial t}=K_{i}(x)+J_{i}(x-1, t)-J_{i}(x, t),
\end{aligned}
$$




$$
\sum_{x=2}^{\infty} x K_{v}(x)=\varepsilon_{v} G_{N R T}\left(1-\varepsilon_{r}\right), \sum_{x=2}^{\infty} x K_{i}(x)=\varepsilon_{i} G_{N R T}\left(1-\varepsilon_{r}\right)
$$

The $K_{v}(x), K_{i}(x)$ are the rates of in-cascade generation of voids and SIA loops, respectively, $\varepsilon_{r}$ is the fraction of Frenkel pair which recombine during the cascade cooling, $\varepsilon_{v}, \varepsilon_{i}$ are the incascade clustering fractions for vacancies and SIAs, respectively, $G_{N R T}$ is the NRT Frenkel pair generation rate, and $J_{v}(x, t), J_{i}(x, t)$ are the void and SIA loop fluxes in cluster size space, respectively:

$$
\begin{gathered}
J_{v}(x, t)=P_{v}(x, t) f(x, t)-Q_{v}(x+1, t) f(x+1, t), \\
J_{i}(x, t)=P_{i}(x, t) f(x, t)-Q_{i}(x+1, t) f(x+1, t) .
\end{gathered}
$$

In Eqs. (2), $P_{v}(x, t)$ and $P_{i}(x, t)$ are the rates of vacancy absorption by a void and SIA absorption by a SIA cluster, respectively; $Q_{v}(x, t)$ is the sum of the rates of SIA absorption and vacancy emission from a void, and $Q_{i}(x, t)$ the rate of vacancy absorption by a SIA cluster. These rates depend on the cluster concentration and the diffusion properties of the mobile defects, i.e. vacancies and SIAs. In the case of 3-D diffusion of point defects to voids and SIA loops the rates take the following form $[4,11]$ :

$$
\begin{gathered}
P_{v}(x)=w_{v} x^{1 / 3} D_{v} C_{v}(t), \\
Q_{v}(x)=w_{v} x^{1 / 3}\left[D_{i} C_{i}+D_{v} \exp \left(-E_{v}^{b}(x) / k T\right)\right]=Q_{v}^{i}(x)+Q_{v}^{v}(x), \\
P_{i}(x)=Z_{i}^{l} w_{i} x^{1 / 2} D_{i} C_{i}(t), \\
Q_{i}(x)=Z_{v}^{l} w_{i} x^{1 / 2} D_{v} C_{v}(t), \\
w_{v}=\left(\frac{48 \pi^{2}}{\Omega^{2}}\right)^{1 / 3}, w_{i}=\left(\frac{4 \pi}{\Omega b}\right)^{1 / 2} .
\end{gathered}
$$

$C_{i, v}(t)$ and $D_{i, v}$ are the concentrations and diffusion coefficients, respectively, of vacancies (subscript v) and SIAs (subscript i), $Z_{v}^{l}, Z_{i}^{l}$ are the dislocation loop capture efficiencies for vacancies and SIAs, $E_{v}^{b}(x)$ is the binding energy of a vacancy to a vacancy cluster of size $\mathrm{x}, k_{B}$ is Boltzmann's constant, $\mathrm{T}$ is the absolute temperature, $\Omega$ is the atomic volume, and $\mathrm{b}$ is the magnitude of the SIA loop Burgers vector. In the case when the dislocation loops are treated as 3 -D clusters, which is used in the OKMC calculations, the parameter $w_{i}$ has to be replaced by $w_{v}$ and $x^{1 / 2}$ replaced by of $x^{1 / 3}$ in Eqs. (4a) and (4b). 
Note that if the SDFs described by Eqs. (1a) and (1b) are written in x-space, the cluster densities are dimensionless. That is, the total density of clusters, $N_{v, i}=\sum_{x=2}^{\infty} f_{v, i}(x)$, has the units [1/atom]. The capture efficiencies used in Eqs. (3) and (4) have dimensions of $\left[\mathrm{m}^{-2}\right]$, and the cluster sink strengths, which are given by:

$$
\begin{aligned}
& k_{v}^{2}=\sum_{x=2}^{\infty} P_{v}(x) f_{v}(x) \equiv w_{v} \sum_{x=2}^{\infty} x^{1 / 3} f_{v}(x), \\
& k_{l(v, i)}^{2}=\sum_{x=2}^{\infty} P_{i}(x) f_{i}(x) \equiv Z_{v, i}^{l} w_{i} \sum_{x=2}^{\infty} x^{1 / 2} f_{i}(x),
\end{aligned}
$$

also have the dimensionality of $\left[\mathrm{m}^{-2}\right]$. For the purpose of comparing the results obtained by the RT and OKMC techniques, it will be useful to show that the sink strengths given by Eqs. (6) are equivalent to those that are normally used in the mean size approximation in RT models:

$$
\begin{aligned}
& k_{v}^{2}=4 \pi<R_{v}>N_{v}, \\
& k_{l(v, i)}^{2}=2 \pi Z_{v, i}^{1}<R_{i}>N_{i},
\end{aligned}
$$

which describe damage accumulation in the form of voids and loops using the loop capture efficiency for vacancies and interstitials, $Z_{v, i}^{l}$, the mean cluster radii $\left\langle\mathrm{R}_{v}\right\rangle$ and $\left\langle\mathrm{R}_{i}>\right.$, and the corresponding total number densities $N_{v}$ and $N_{i}$ with units of $m$ and $m^{-3}$, respectively. In order to show this, the SDF can be calculated in the domain where the size of a cluster is defined by its radius, $r$, instead of $\mathrm{x}$. The SDFs can be calculated by taking into account that a sum of the SDF over all sizes in any phase space used for the ME has to be equal to the total number of clusters, $N_{\text {tot }}$. Replacing the sums with integrals, the total number density of the clusters in the case under consideration may be written as

$$
N_{\text {tot }}=\int_{x=2}^{\infty} f(x) d x \equiv \int_{R=R_{\min }}^{\infty} f(R) d R
$$

The two integral can be equal to each other if

$$
f(x) d x=f(R) d R .
$$

Taking into account that the radii of voids, $r_{v}$, and loops, $r_{i}$, and the total number of defects in the clusters, $x_{v}, x_{i}$, related to each other as follows

$$
\frac{4 \pi}{3} R_{v}^{3}=x_{v} \Omega, \pi R_{i}^{2} b=x_{i} \Omega,
$$


and using Eq. (9) the functions $f_{v}\left(r_{v}\right)$ and $f_{i}\left(r_{i}\right)$ can be easily calculated

$$
f_{v}\left(R_{v}\right)=f_{v}(x)\left(\frac{36 \pi}{\Omega}\right)^{1 / 3} x^{2 / 3}, \quad f_{i}\left(R_{i}\right)=f_{i}(x)\left(\frac{4 \pi b}{\Omega}\right)^{1 / 2} x^{1 / 2} .
$$

Note that dimensionality of the functions $f_{v}\left(R_{v}\right), f_{i}\left(R_{i}\right)$ is $\left[\frac{1}{m} \frac{1}{a t o m}\right]$. On the other hand the SDFs in r-space are normally presented as a number of clusters per unit volume. This can be obtained by dividing the right hand sides of Eqs. (11) by the atomic volume, $\Omega$, i.e. $F_{v, i}\left(R_{v, i}\right)=\frac{1}{\Omega} f_{v, i}\left(R_{v, i}\right)$. Here the capital $\mathrm{F}$ is used to distinguish between the dimensional SDF

$\left(1 / \mathrm{m}^{4}\right)$ and SDFs $\left.f_{v}\left(R_{v}\right), f_{i}\left(R_{i}\right)\right)$. Using Eqs. (10), (11) and replacing the sums in Eq. (6) by the corresponding integrals one can easily find that:

$$
\begin{gathered}
k_{v}^{2}=\left(\frac{48 \pi^{2}}{\Omega^{2}}\right)^{1 / 3} \int_{x=2}^{\infty} x^{1 / 3} f_{v}(x) d x=4 \pi \int_{R_{v, \text { min }}}^{\infty} R_{v} F_{v}\left(R_{v}\right) d R_{v} \equiv 4 \pi<R_{v}>N_{v}, \\
k_{i(v, i)}^{2}=Z_{v, i}^{l}\left(\frac{4 \pi}{\Omega b}\right)^{1 / 2} \int_{x=2}^{\infty} x^{1 / 2} f_{i}(x)=2 \pi Z_{v, i}^{l} \int_{R_{i, \text { min }}}^{\infty} R_{i} F_{i}\left(R_{i}\right) d R_{i} \equiv 2 \pi Z_{v, i}^{l}<R_{i}>N_{i}
\end{gathered}
$$

where

$$
<R_{v}>=\frac{\int_{R_{v, \text { min }}}^{\infty} R_{v} F_{v}\left(R_{v}\right) d R_{v}}{\int_{R_{v, \text { min }}}^{\infty} F_{v}\left(R_{v}\right) d R_{v}}, \quad<R_{v}>=\frac{\int_{R_{i, \text { min }}}^{\infty} R_{i} F_{i}\left(R_{i}\right) d R_{i}}{\int_{R_{i, \text { min }}}^{\infty} F_{i}\left(R_{i}\right) d R_{i}} .
$$

Although the integral of the SDFs shown in Eq. (7) has the same value for either phase space, the difference in dimensionality means the two SDF have quite different shapes in $\mathrm{r}$ - or $\mathrm{x}$ space. This is shown in Fig. 1, where r- and x-space SDFs calculated using the RT for a specific case are compared. Although use of the $\mathrm{x}$-space description may be more convenient for purposes of the calculations, the r-space SDF is more appropriate for comparing with experimental observations such as TEM measurements. 


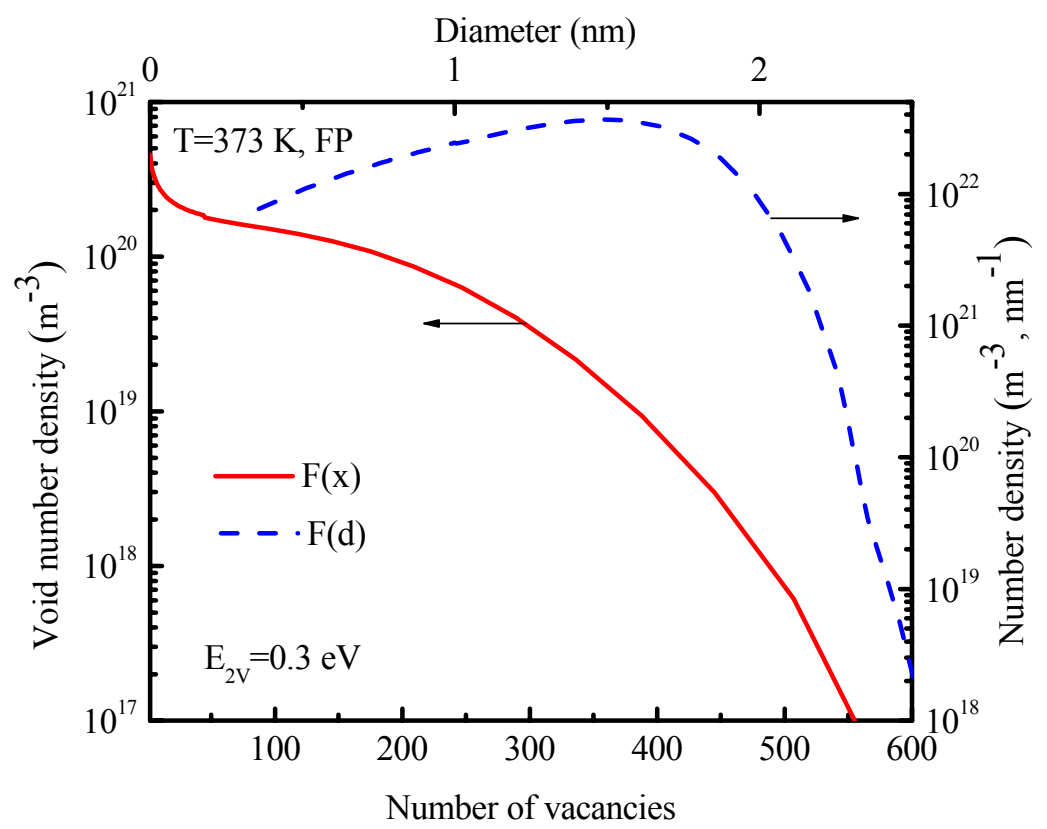

Fig. 1. Void size distribution function calculated for the case of $E_{2 v}=0.3: F(x)$ in phase space $x$ ( $x=$ number of vacancies), and $F(d)$ in phase space $d$ ( $d=$ void diameter).

\subsubsection{Initial and boundary conditions}

The initial and boundary conditions for SDFs of void, SIA loops and point defect concentrations are taken to have the following form

$$
\begin{gathered}
f_{v}(x, t=0)=C_{v 0} \delta(x-1), \quad f_{i}(x, t=0)=0, \quad(x \geq 1), \\
f_{v}(x=1, t)=C_{v}(t), \quad f_{i}(x=1, t)=C_{i}(t), \\
f_{v}(x=\infty, t)=f_{i}(x=\infty, t)=0, \\
C_{v}(t=0)=C_{v 0}, \quad C_{i}(t=0)=0,
\end{gathered}
$$

where $C_{v 0} \exp \left(-\frac{E_{v}^{f}}{k_{B} T}\right)$ is the thermal equilibrium vacancy concentration $\left(E_{v}^{f}\right.$ is the vacancy formation energy) and $\delta(x)$ is the Kronneker delta.

\subsubsection{Equations for point defect concentrations}

In order to complete the system of equations, we must add equations for the evolution of the vacancies, $C_{v}$, and SIAs, $C_{i}$, which are given by [6]: 


$$
\begin{gathered}
\frac{d C_{v}(t)}{d t}=G_{N R T}\left(1-\varepsilon_{r}\right)\left(1-\varepsilon_{v}\right)-\left[\mu_{R} D_{i} C_{i}(t) C_{v}(t)+Z_{v}^{d} \rho D_{v}\left(C_{v}(t)-C_{v 0}\right)+\sum_{x=2}^{x=\infty} P_{i}(x) f_{i}(x, t)\right] \\
-\left[\left(P_{v}(1) f_{v}(1, t)-Q_{v}^{v}(2) f_{v}(2, t)\right)-P_{i}(2) f_{v}(2, t)\right]-\sum_{x=1}^{x=\infty}\left(P_{v}(x) f_{v}(x, t)-Q_{v}^{v}(x+1) f_{v}(x+1, t)\right) \\
\frac{d C_{i}(t)}{d t}=G_{N R T}\left(1-\varepsilon_{r}\right)\left(1-\varepsilon_{i}\right)-\left[\mu_{R} D_{i} C_{i}(t) C_{v}(t)+Z_{i}^{d} \rho D_{i} C_{i}+\sum_{x=1}^{x=\infty} P_{i}(x) f_{i}(x, t)\right] \\
-\left[P_{i}(1) f_{i}(1, t)-P_{v}(2) f_{i}(2, t)\right]-\sum_{x=1}^{x=\infty} Q_{v}^{i}(x+1) f_{v}(x+1, t)
\end{gathered}
$$

where $G_{N R T}$ is the point defect generation rate, $\mu_{R}$ is the recombination coefficient, $\rho$ is the dislocation density, and $Z_{v}^{d}, Z_{i}^{d}$ are the dislocation capture efficiencies for vacancies and SIAs, respectively (here $Z_{v}^{d}$ is set equal to 1.0). The first term on the right hand side of Eqs. (15) and (16) represents the generation rates of the mobile point defects, and the second term represents the capture rates by mutual point defect recombination, dislocations and the SIA loops. The third term accounts for the fact that reactions between two vacancies and two SIAs eliminate both of them at the same time, and the generation of vacancies and SIAs by reactions between a di-vacancy and SIA and di-SIA and vacancy. The last term represents the point defect capture rates by voids.

Eqs. (1)-(4) and(15), (16) comprise a large system of coupled nonlinear differential equations and, in the general case, can be only solved by numerical methods. For realistic sizes of voids and SIA clusters the system size can exceed $10^{6}$ equations, requiring that practicable solution of this system will require some grouping procedure to minimize the number of equations. This amounts to approximating the continuous SDF as a histogram. In the present work the grouping method developed by Golubov, et al. [6] was used.

\subsection{Object kinetic Monte Carlo Model}

\subsubsection{Thermally-activated processes}

The general features of the OKMC code used in the present work, LAKIMOCA, are described in [12]. The model treats radiation produced defects (vacancies, self-interstitials atoms, and clusters thereof) as objects that have specific reaction volumes and that are located in particular positions in the simulation box. Each object can migrate and participate in a series of 
predefined reactions. The probabilities for physical transition mechanisms, which are basically migration jumps and emission from larger defects or from traps, are calculated in terms of Arrhenius frequencies for thermally activated events, $\Gamma_{i}=v_{i} \exp \left(-\frac{E_{a, i}}{k_{B} T}\right)$, where $v_{i}$ is the attempt frequency (pre-factor) for event $i$, and $E_{a, i}$ is the corresponding activation energy. The Monte Carlo algorithm [9] is used at each step to select the event that is going to take place, based on the corresponding probabilities. After a certain event is chosen, time is increased according to the residence time algorithm, $\Delta \tau=\left(\sum_{i=1}^{N_{e}^{\text {th }}} \Gamma_{i}+\sum_{j=1}^{N_{e}^{\text {eet }}} P_{j}\right)^{-1}[13]$, where the $P_{j}$ are the probabilities of external events, such as the appearance of a cascade or of isolated Frenkel pair produced by impinging particles. The choice of this expression is in the long term equivalent to choosing $\Delta \tau^{\prime}=-\ln R^{*} \Delta \tau$, where $\mathrm{R}$ is a random number between 0 and 1 [14].

\subsubsection{Sink strengths in the OKMC}

In addition, the model includes non-thermally activated events, such as the annihilation of a defect after encountering either a defect of opposite nature (i.e. a SIA encountering a vacancy) or a sink, as well aggregation, either by adding a point-defect to a cluster or by forming a complex between a defect and a trap for it. These events occur only on the basis of geometrical considerations (overlap of reaction volumes) and do not participate in defining the progression of time. It is possible to introduce different classes of immobile traps and sinks, characterized by specific geometrical shapes (spheres, infinite cylinders, surfaces etc.) suitable for mimicking voids and other defects such as dislocations and grain boundaries.

In the present work, the OKMC technique is used to simulate damage accumulation in the according to the framework of the model formulated above. A bcc iron lattice containing edge dislocations is simulated, with the evolution of two types of point defect clusters, voids and SIA loops. To simplify the calculations, both types of defect clusters are treated as spherical absorbers. The only difference between the vacancy clusters and the SIA loops is that the first ones are described as neutral sinks, whereas the second are considered to be biased, i.e. having preference for absorption of SIAs. Thus, instead of using Eq. (6b), the sink strength of the SIA clusters is given by: 


$$
k_{i(v, i)}^{2}=Z_{v, i}^{l} w_{v} \sum_{x=2}^{\infty} x^{1 / 3} f_{i}(x) \equiv 4 \pi Z_{v, i}^{l}<R_{i}>N_{i}, \quad\left(Z_{v}^{l}=1, Z_{i}^{l}>1\right) .
$$

The bias of the SIA clusters is equal to $p_{l}=\left(Z_{i}^{l}-Z_{v}^{l}\right) / Z_{v}^{l} \equiv Z_{i}^{l}-1$ and in the following calculations is chosen to be equal of that for the dislocations, i.e. $Z_{i}^{l}=Z_{i}^{d}$. Note that such an approach for the shape of SIA clusters in the following calculations is quite reasonable since the sink strengths of small spherical clusters and that of dislocation loops are similar when the cluster size is not large. Using Eqs. (6) one can find that the ratio of the sink strengths is given by

$$
\frac{\left(k_{i}^{2}\right)_{\text {sperical }}}{\left(k_{i}^{2}\right)_{\text {loop }}} \approx\left(\pi 3^{3 / 2}\right)^{1 / 6} x^{-1 / 6}=1.592 x^{-1 / 6}
$$

i.e. it only weakly depends on the number of defects in the cluster (it varies within a factor 2 when $x$ varies in a range from 2 to $10^{4}$ ). This size range matches well the calculations presented in this work since the highest irradiation dose used in the calculations is rather small $\left(10^{-2} \mathrm{dpa}\right)$.

When the sink strength of small clusters is accounted for in the OKMC, a measurable difference is observed between the RT and OKMC. This arises from the discrete spatial description of point defect absorption in the OKMC. In the case of the RT, the equivalent radius of a cluster containing any number of point defects can be calculated using Eq. (10), and this radius determines the sink strength, which is essentially the probability of the cluster absorbing a mobile point defect in that model. However, in the OKMC model, a cluster of $X$ point defects occupies $X$ lattice sites. Point defect absorption occurs when a mobile point defect arrives at an adjacent lattice site. For relatively large clusters the reaction probability or sink strength is nearly the same since the larger clusters are more nearly spherical. However, the equivalent radius given by Eq. 10 over-estimates the reaction rate for small clusters. This is illustrated in Fig. 2, in which the sink strength obtained by the two models is shown for vacancy clusters containing up to 500 vacancies (equivalent radius of $1.12 \mathrm{~nm}$ ). The discrete points were obtained by OKMC simulations in which point defect absorption was computed for vacancy clusters of a the indicated size. The lines indicate RT sink strengths computed using the equivalent radius from Eq. (10). The dashed line is obtained using the lowest order expression for the sink strength, Eq. (7a), and the solid line is obtained when the first order correction term is included to account for multiple sink effects [15]. This multiple sink correction is implicitly accounted for in the OKMC 
method. The impact of this difference in sink strength will be discussed further when the results are presented in Section 3.

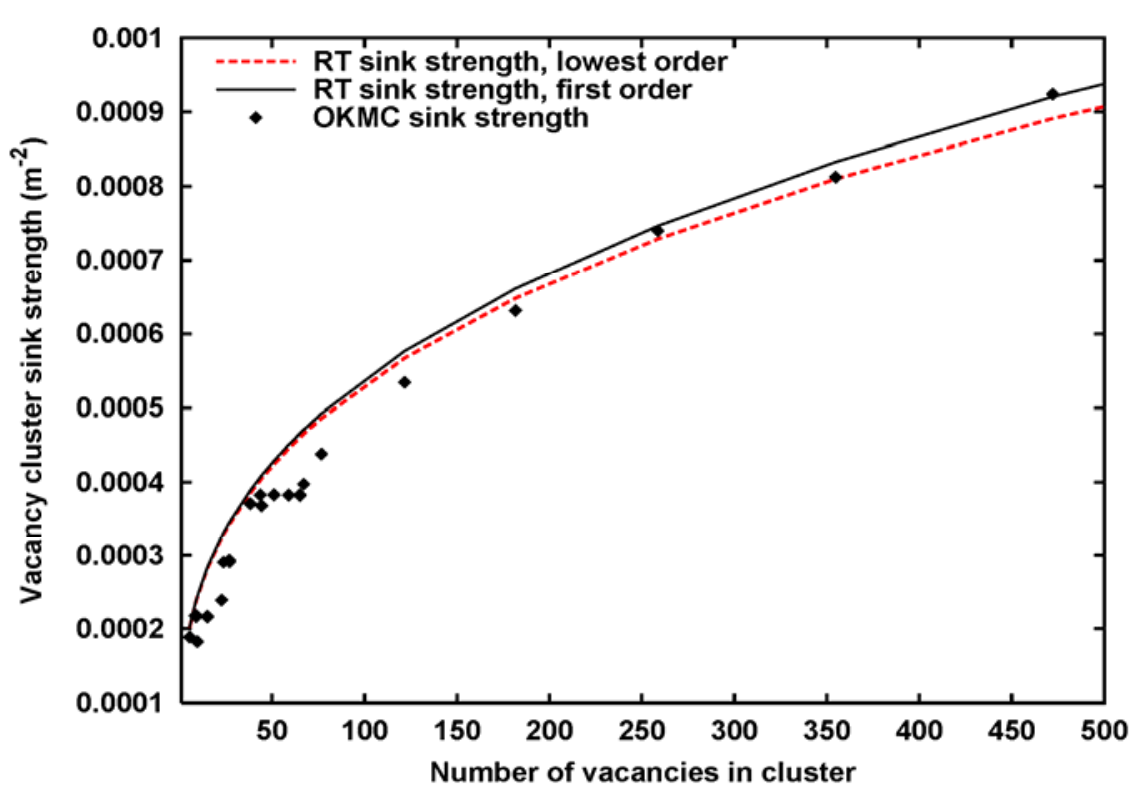

Fig. 2. Comparison of vacancy cluster sink strength obtained in OKMC simulations (discrete points) and the analytical expression applied in the RT (lines). The dashed line is the lowest order sink strength, which is valid for small sink volume fractions, and the solid line includes the first order sink strength correction factor [15].

More than one approach could be followed to simulate the sink strength of dislocations in OKMC. One possible method is the direct introduction of an absorbing cylinder of a certain length, $L_{d}$, corresponding to the desired dislocation density, $\rho$, and with specified capture efficiencies for point defects. In this case, one may expect to reproduce the sink strength given in Eqs. (15) and (16) with the efficiencies $Z_{v, i}^{d}=\frac{2 \pi}{\ln \left[\left(k R_{c v, c i}\right)^{-1}\right]}$, which leads to a dislocation bias to be equal to:

$$
p=\frac{Z_{i}^{d}-Z_{v}^{d}}{Z_{v}^{d}}=\frac{\ln \left(R_{d i} / R_{d v}\right)}{\ln \left[\left(k R_{d i}\right)^{-1}\right]}
$$

where $r_{d i}, r_{d v}$ are the cylinder capture radii for SIAs and vacancies, respectively, and $k=\sqrt{k^{2}}$ (where $k^{2}$ is the total sink strength in the crystal). This approach appears relatively 
intuitive and straight forward, but this is not the case. The first problem arises with choosing the cylinder length. In order to simulate a given dislocation density, $\rho$, the length of the cylinder has to be taken to be equal to $L_{d}=\rho V_{b o x}$, which may be too small or too large to fit conveniently in the simulation box, $L_{b o x}=V_{b o x}{ }^{1 / 3}$. In addition, the dislocation density can vary by several orders of magnitude whereas the maximum box size is essentially fixed by computational limitations. As a result, such a cylinder may have its ends inside the simulation box, and thus cannot reproduce the actual cylindrical symmetry of a dislocation. The impact of this on the dislocation sink strength requires further investigation, which is beyond the scope of the present work, and is not directly relevant to the comparison of the RT and OKMC methods. A second issue arises from the fact that the dislocation sink strength and corresponding bias depends on the total sink strength, $k^{2}$, see Eq. (24), which changes during the irradiation. Because of these issues, the alternate approach discussed in the next paragraph was used to simulate the dislocation sink density.

One way to avoid these complications is to simulate the dislocation sink by introducing another class of spherical absorber, which maintains the two main properties of dislocations as a sink for point defects: (a) a preference for SIA absorption, i.e. maintain a constant dislocation bias, and (b) maintain constant sink strength during irradiation. This may be achieved by introducing additional spherical absorbers with fixed density and size as follows

$$
\begin{gathered}
k_{v d}^{2}=4 \pi R_{v}^{d} N^{d} \equiv \rho, \\
k_{i d}^{2}=4 \pi R_{i}^{d} N^{d}, \quad\left(R_{i}^{d}>R_{v}^{d}\right) .
\end{gathered}
$$

Thus choosing a specific capture radius for vacancies, $R_{v}^{d}$, the density of the absorbers, $N^{d}$, can be calculated from the first Eq. $(25), N^{d}=\frac{\rho}{4 \pi R_{v}^{d}}$. The bias in the case is equal to

$$
p=\frac{k_{i}^{2}-k_{v}^{2}}{k_{v}^{2}}=\frac{R_{i}^{d}}{R_{v}^{d}}-1,
$$

i.e. it depends on the ratio of $R_{i}^{d} / R_{i}^{d}$ only. Taking the ratio $R_{i}^{d} / R_{i}^{d}$ to be equal to $Z_{i}^{d}$ one may expect that Eqs. (25) will reproduce the sink strength of dislocations with respect to their ability to capture point defects. Thus, Eqs. (25) and (26) permit calculating the absorber properties of dislocation at any given dislocation density and bias. The spherical absorbers used 
to simulate dislocations are randomly distributed in the simulation box, with capture radii of $R_{v}^{d}=0.4 \mathrm{~nm}, R_{i}^{d}=0.48 \mathrm{~nm}$ for vacancies and SIAs, respectively,

An OKMC simulation cell size of $300 \times 300 \times 300$ lattice parameters was used in all the simulations discussed here. Given the lattice parameter of $\alpha$-Fe (see Table 2), this leads to a volume equal to $6.423 \times 10^{-22} \mathrm{~m}^{3}$. As a result, the minimum density of any given object that can be obtained in the OKMC simulations is $1.56 \times 10^{21} \mathrm{~m}^{-3}$, i.e. one per box volume. The impact of this limitation will be discussed below.

\subsection{Material and irradiation parameters}

In order to explore a range of irradiation phenomena, and to help isolate the effects of different mechanisms, two types of irradiation conditions were considered:

1. Pure Frenkel pair production only, which is similar to electron irradiation, and

2. Cascade damage production, typical of heavy ion or neutron irradiation, in which $30 \%$ of the SIA and vacancies are produced in small clusters (14\% di-, $12 \%$ tri-, $4 \%$ tetra-interstitials) and 6-vacancy clusters.

For the second case, two classes of cascade debris were considered in the OKMC simulations: (2a) normal cascades in which the point defects and defect clusters were spatially correlated as observed in atomistic simulations of cascade damage formation [16], and (2b) special "random" cascades in which the spatial correlation was not preserved. Case (2b) is a better approximation of how the damage production is simulated in the rate theory model. The cascade efficiency, i.e. the fraction of point defects that survive after cascade cooling phase, is taken to be 0.4 for the cases of cascade damage production. When simulating Frenkel pair production, the cascade efficiency is essentially 1.0 , so the damage rate was set to permit comparison with cascade damage results at the same effective displacement rate.

The binding energy of a vacancy with a void of size $x$ is described using the capillarity model adjusted to a specific di-vacancy binding energy, $E_{2 v}$. The general expression for the vacancy binding energy takes the following form:

$$
E_{v}^{b}(x)=E_{v}^{f}+\left(E_{2 v}-E_{v}^{f}\right)\left(\frac{x^{2 / 3}-(x-1)^{2 / 3}}{2^{2 / 3}-1}\right) .
$$


Table 2. Material and irradiation parameters used in calculations

\begin{tabular}{|l|l|}
\hline Temperature, $\mathrm{T}$ & 373 to $523 \mathrm{~K}$ \\
\hline Lattice parameter, $a$ & $0.2876 \mathrm{~nm}$ \\
\hline Atomic volume, $\Omega=a^{3} / 2$ & $1.189 \times 10^{-29} \mathrm{~m}^{3}$ \\
\hline Number of atoms in unit volume, $N=1 / \Omega$ & $8.407 \times 10^{28} \mathrm{~m}^{-3}$ \\
\hline Box volume of a cube of size $300 \mathrm{a}, V_{b o x}$ & $6.423 \times 10^{-22} \mathrm{~m}^{3}$ \\
\hline Number of atoms in the box, $N_{b o x}=V_{b o x} / \Omega$ & $5.4 \times 10^{7}$ \\
\hline Number density equivalent to one cluster in, $N_{c l}=1 / V_{b o x}$ & $1.557 \times 10^{21} \mathrm{~m}^{-3}$ \\
\hline NRT displacement rate, $G_{N R T}$ & $4 \times 10^{-7}$ to $4 \times 10^{-5} \mathrm{dpa} / \mathrm{s}$ \\
\hline Cascade survival efficiency, $\left(1-\varepsilon_{r}\right)$ & 0.40 \\
\hline Fraction of SIAs in cluster form, $\varepsilon_{i}(\mathrm{x}=2,3$ and 4$)$ & 0.30 \\
\hline Fraction of voids in cluster form, $\varepsilon_{v}(\mathrm{x}=6)$ & 0.30 \\
\hline Recombination coefficient, $\mu_{R}=4 \pi\left(r_{v}+r_{i}\right) / \Omega,\left(r_{v}+r_{i}=0.4466 \mathrm{~nm}\right)$ & $4.72 \times 10^{20} \mathrm{~m}^{-2}$ \\
\hline Attempt frequency, $v$ & $6.0 \times 10^{12} \mathrm{~s}^{-1}$ \\
\hline $\begin{array}{l}\text { Vacancy diffusion coefficient, } D_{v}=D_{v 0} \exp \left(-\frac{E_{v}^{m}}{k T}\right) \\
\text { pre-exponential, } D_{v 0}=\frac{1}{6} r^{2} v,\left(r=\frac{\sqrt{3}}{2} a\right)\end{array}$ & $6.02 \times 10^{-8} \mathrm{~m}^{2} / \mathrm{s}$ \\
\hline migration energy, $E_{v}^{m}$ & $0.65 \mathrm{eV}$ \\
\hline SIA diffusion coefficient, $D_{i}=D_{i 0} \exp \left(-\frac{E_{i}^{m}}{k T}\right)$ & $0.2-0.8 \mathrm{eV}$ \\
\hline $\begin{array}{l}\text { pre-exponential, } D_{i 0}=\frac{1}{6} r^{2} v \\
\text { migration energy, } E_{i}^{m}\end{array}$ & $1.0,1.20$ \\
\hline Binding energy of di-vacancies, $E_{2 v}$ & $3 \times 10^{14} \mathrm{~m}^{-2}$ \\
\hline Capture efficiencies of dislocations and SIA clusters, $Z_{v}^{l}, Z_{i}^{l}$ & $0.48 \mathrm{and} 0.4 \mathrm{~nm}$ \\
\hline Dislocation density, $\rho_{d}$ & $6.2)$ \\
\hline Capture radii for “dislocation" spherical absorbers, $R_{i}^{d}, R_{v}^{d}$ & $0.30 \mathrm{eV} 0^{-8} \mathrm{~m}^{2} / \mathrm{s}$ \\
\hline
\end{tabular}

The energy $E_{2 v}$ can be treated as a variable parameter to permit investigation of the 
stability of the vacancy clusters. The same value is applied in both the OKMC and RT models. The SIA clusters are treated as thermally stable. The nominal material and irradiation parameters are given in Table 2 .

\section{Results}

A large number of figures will be presented to summarize the primary results. A consistent color scheme has been applied in each of the figures to aid the reader. The RT results are shown as red curves, and the OKMC as black symbols or curves. Parameters that were varied in the analysis are differentiated by different types of lines. In some cases, two sets of OKMC results are shown at a given dose. One is the instantaneous value at that dose, and the other is the average value obtained from that dose and the previous four timesteps. This helps to demonstrate the statistical nature of the OKMC results and permits results to be shown when the average value is less than the minimum of one per OKMC box volume. This minimum density is also indicated in some of the figures. The results presented include calculated values of: vacancy and interstitial concentrations, vacancy and SIA cluster densities, the total number of vacancies and SIA accumulated in clusters, and the vacancy and SIA cluster size distributions. The net number of point defects accumulated in clusters is significant because it represents the volume change or swelling associated with the irradiation.

The simplest situation to simulate is that of only Frenkel pair production, with vacancy and interstitial cluster formation occurring only as a result of homogeneous nucleation. For this case, the three different sink behavior possibilities were considered:

(1) no fixed sinks, vacancy and interstitial clusters can form by classical nucleation (diffusive encounters), the capture efficiency of both cluster types for mobile mono-vacancies and mono-interstitials is the same, i.e. there is no biased absorption;

(2) the same as in (1) above, but SIA clusters have $20 \%$ interstitial bias; and

(3) the same as in (2) above but the fixed (dislocation) sink with an interstitial bias of $20 \%$ and a density of $3 \times 10^{14} \mathrm{~m}^{-2}$ is included.

Of these three, the last one is certainly most physically representative of the behavior expected in real materials. The use of the other two was intended to be a tool for isolating the effect of specific point defect / sink reactions to aid in the comparison and evaluation of the two 
computational models.

The results of RT and OKMC simulations for these conditions are shown in Figs. 3-7 for an irradiation temperature of $373 \mathrm{~K}$ and an NRT displacement rate of $4 \times 10^{-7} \mathrm{dpa} / \mathrm{s}$. The dose dependence of the vacancy concentration is shown in Fig. 3, where the expected influence of the various sink structures can be seen. There is initially little difference between the biased and unbiased interstitial clusters cases when point defect clusters are the only sink; the difference increases at higher doses when the cluster sink strength increases. The addition of a biased dislocation sink increases the vacancy concentration at the lowest doses due to increased partitioning of vacancies and interstitials, but the concentration is reduced at higher doses due to a higher overall sink strength.

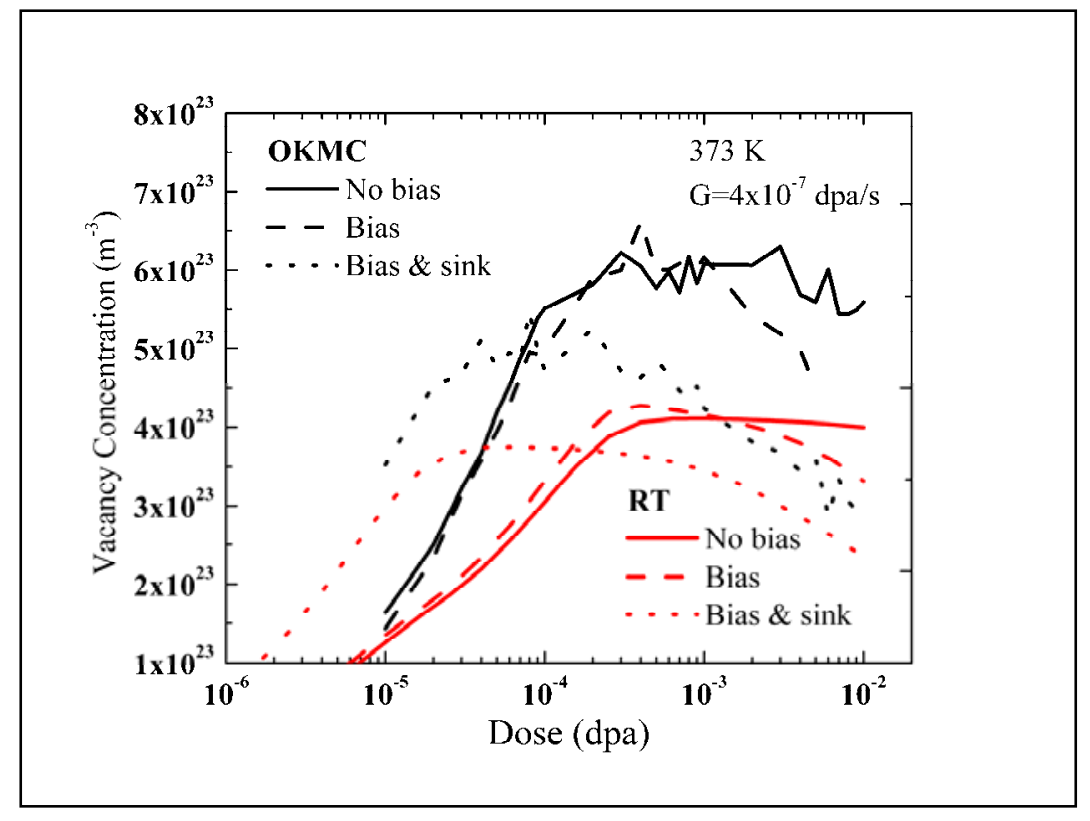

Fig 3. Vacancy concentration for the case of only Frenkel pair production and three sink variants (see text).

Although the trends are similar in both the OKMC and RT results, there is a consistent trend for the vacancy concentration to be higher in the OKMC simulations. This difference arises as a result of the differences in the point defect cluster sink strength discussed above and illustrated in Fig. 2. The lattice-based sink strength obtained with the OKMC is smaller than that from the continuum RT model in the size range of most of the clusters that are generated. This reduced sink strength leads to a higher concentration of free vacancies. Note that difference 
between the two methods is reduced when the dislocation sink is added since the significance of the cluster sink strength is reduced in this case.

The dose dependence of the vacancy and SIA cluster densities are shown in Fig. 4 for the three sink variants. The RT and OKMC predictions of the vacancy concentration are in good agreement for each case. However, the RT results indicate that the interstitial cluster density is near or below the one defect per box value. As a result, the OKMC results are quite variable, ranging between 0 and about 4 per box $\left(6 \times 10^{21} \mathrm{~m}^{-3}\right)$. Limited statistics is also responsible for the irregular time dependence observed in the OKMC curves for vacancy clusters. The simulation cell generally contains less than 10 vacancy clusters even though the displacement rate is relatively high, and the temperature quite low.

The impact is limited statistics is even more clearly observed in the void size distributions shown in Fig. 5. The irradiation and sink conditions remain the same as in Fig. 4, and the density of one defect cluster per OKMC box is indicated. The void SDF obtained from the RT model is smooth and continuous for each of the sink conditions shown in Figs. 5 (1-c). However, the density at any one size is less than one void per unit OKMC box volume. Therefore, the instantaneous density predicted by the OKMC model tends to be either zero or one (or occasionally two) per box volume. Averaging the OKMC results over five timesteps begins to give the appearance of a size distribution value, but the meaning of these values would be difficult to interpret in the absence of the RT results and the knowledge that they represent only one or two voids. The same situation is observed for the interstitial SDF in Figure 6. The RT model produces a smooth SDF for each of the three sink conditions, with the details appropriately dependent on the defect partitioning balance induced by the neutral or biased SIA clusters, and the overall density reduced by the addition of a dominant dislocation sink. At each size, the equivalent density is much less than one per OKMC box volume. As a result, the corresponding OKMC simulations can not reproduce a reasonable approximation of either the absolute SDF, or the variation with sink conditions. Averaged OKMC points are clustered at small sizes with a density between 0.1 and 1.0 per box volume.

The number of defects accumulated in point defect clusters is shown Fig. 7 as a function of dose for the three sink conditions. This value is significant because it represents a net change in the irradiated microstructure that can ultimately be experimentally verified. For example, 

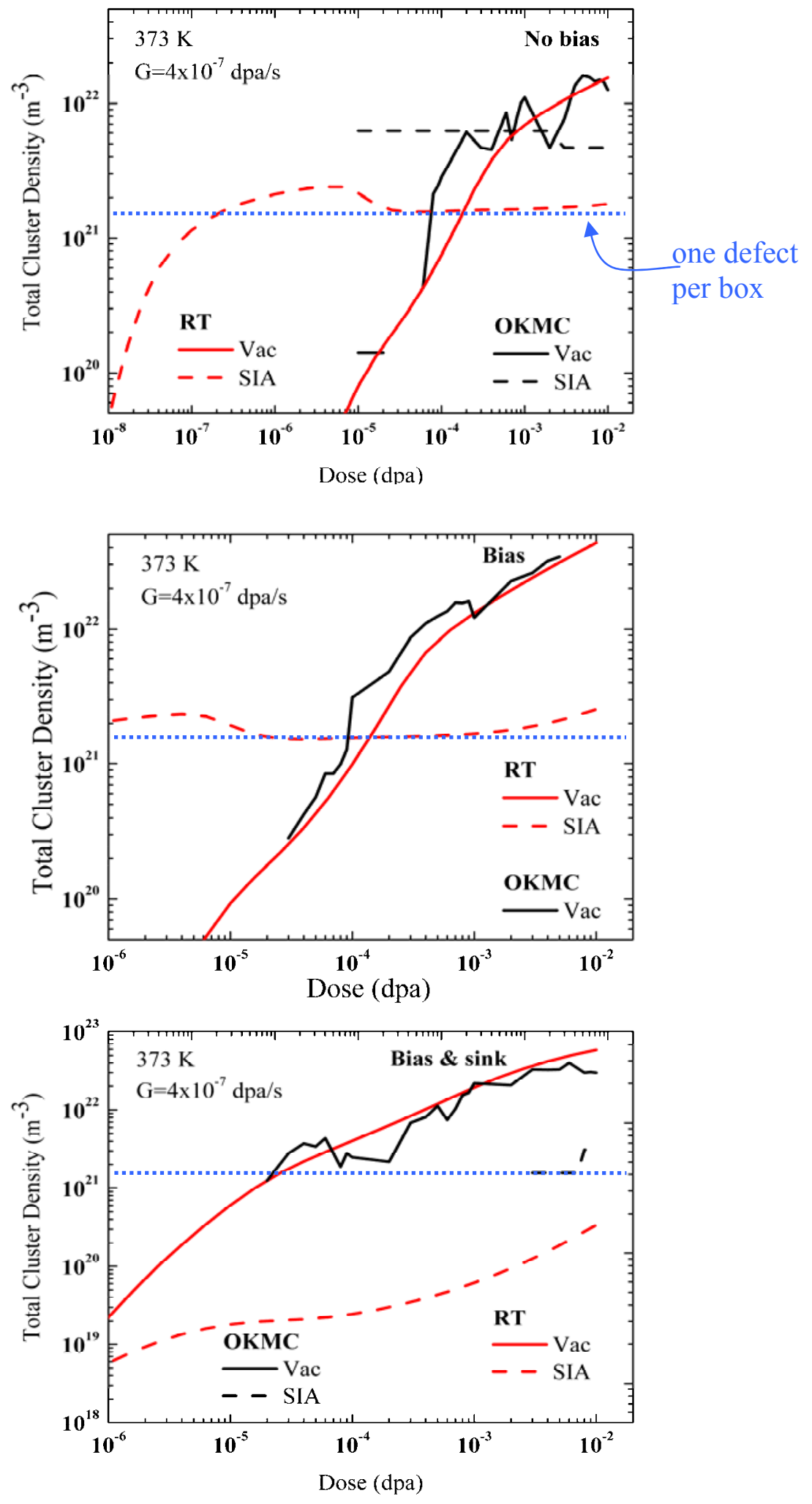

Fig. 4. Dose dependence of vacancy and interstitial cluster density predicted by RT and OKMC for three types of sink structures: (a) only point defect clusters, no biased absorption, (b) same as (a) but interstitial clusters have $20 \%$ bias for SIA, and (c) same as (b) but add fixed dislocation density with $20 \%$ bias for SIA. 

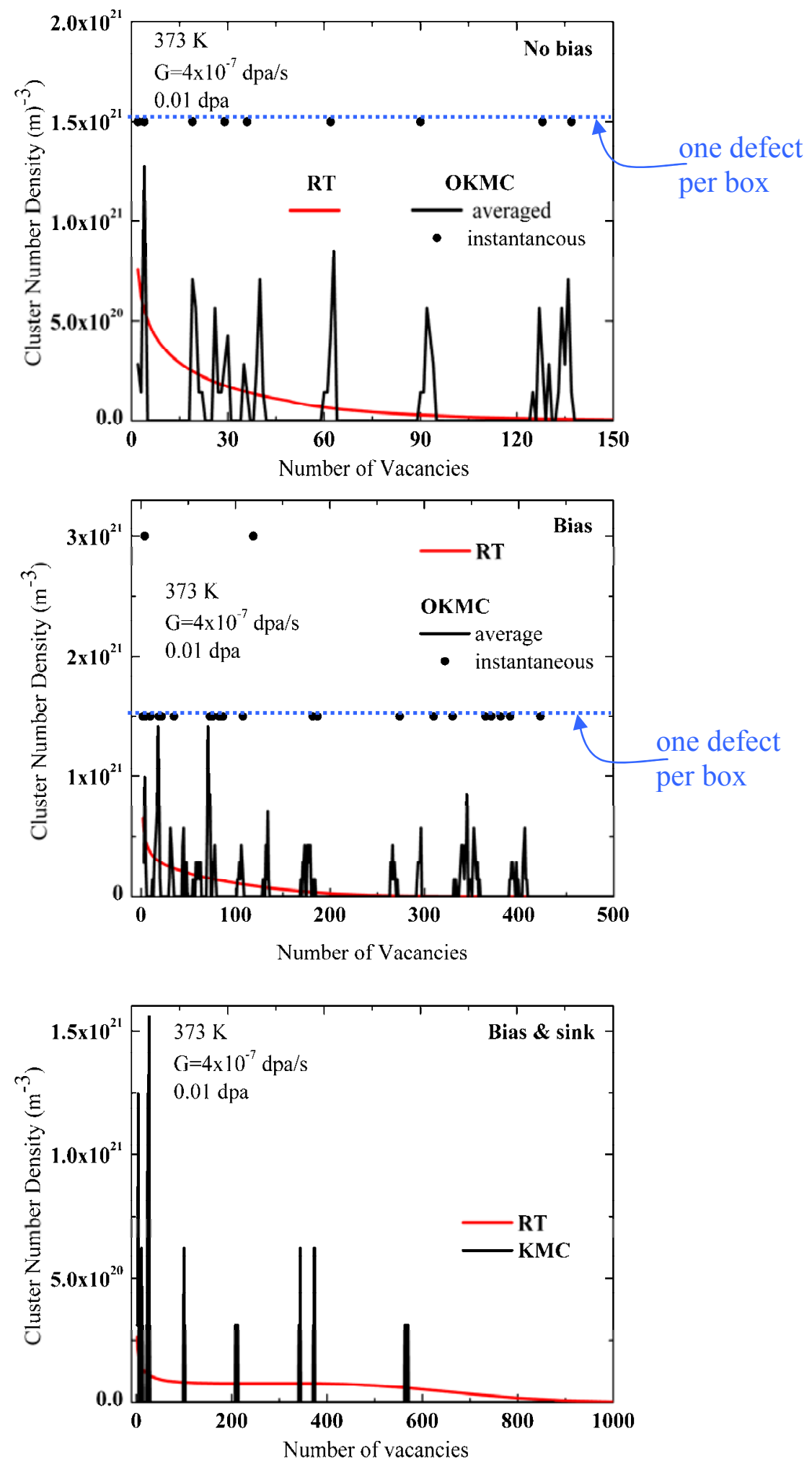

Fig. 5. Vacancy cluster size distributions predicted by RT and OKMC for three types of sink structures: (a) only point defect clusters, no biased absorption, (b) same as (a) but interstitial clusters have $20 \%$ bias for SIA, and (c) same as (b) but add fixed dislocation density with $20 \%$ bias for SIA. 


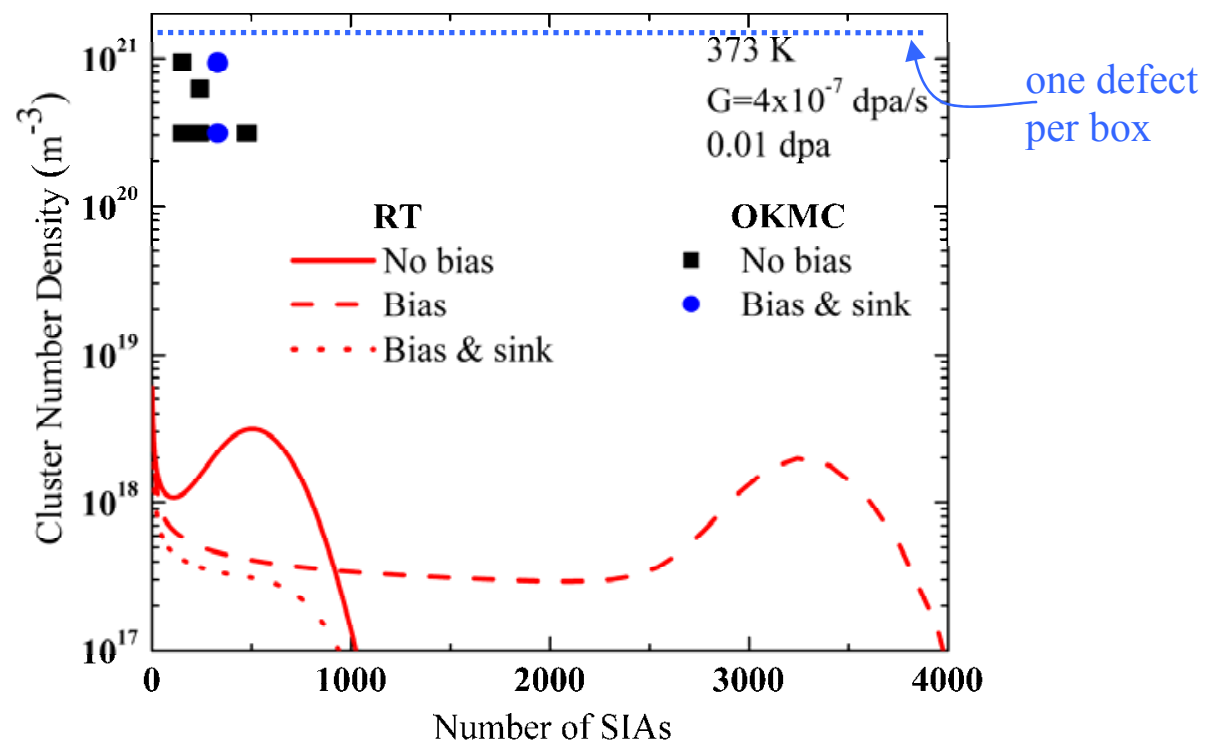

Fig. 6. Interstitial cluster size distributions predicted by RT and OKMC for the same three types of sink structures as in Fig. 5.

volumetric swelling is directly related to accumulation of vacancies. There is good agreement between the RT and OKMC predictions of vacancy accumulation for all three cases, although the dose dependence of the OKMC results are irregular in Figs. 7 (a) and (b) due to the vacancy cluster density being only slightly greater than the one per box value (see Fig. 4). As they should, the total number of vacancies and interstitials accumulated in clusters approach the same value as the dose increases in Figs. 7 (a) and (b). SIA accumulation is also in reasonable agreement except for the third case in Fig. 7(c), where the interstitial cluster density is less than one per OKMC box volume (Fig. 4).

The results shown in Figs. 8 and 9 illustrate the effect of irradiation temperature at a relatively high damage rate of $4 \times 10^{-5} \mathrm{dpa} / \mathrm{s}$. The vacancy concentration is shown in Fig. 8(a) and the void number density in 8(b). Fig. 9(a) shows the number of vacancies accumulated in clusters, and the vacancy cluster size distribution is shown in Fig. 9(b) at two doses for a temperature of $100^{\circ} \mathrm{C}$. The In Figs. 8(a) and (b), and 9(a) the sink conditions include an interstitial bias for the interstitial clusters, but no fixed sink. The fixed dislocation sink is added in Fig. 9(b). The RT and $\mathrm{OKMC}$ results are in reasonable agreement, with the vacancy concentration slightly higher for the OKMC model because of the difference in the vacancy cluster sink strength discussed above. For temperatures greater than about $200^{\circ} \mathrm{C}$, the predictions of the OKMC model show 

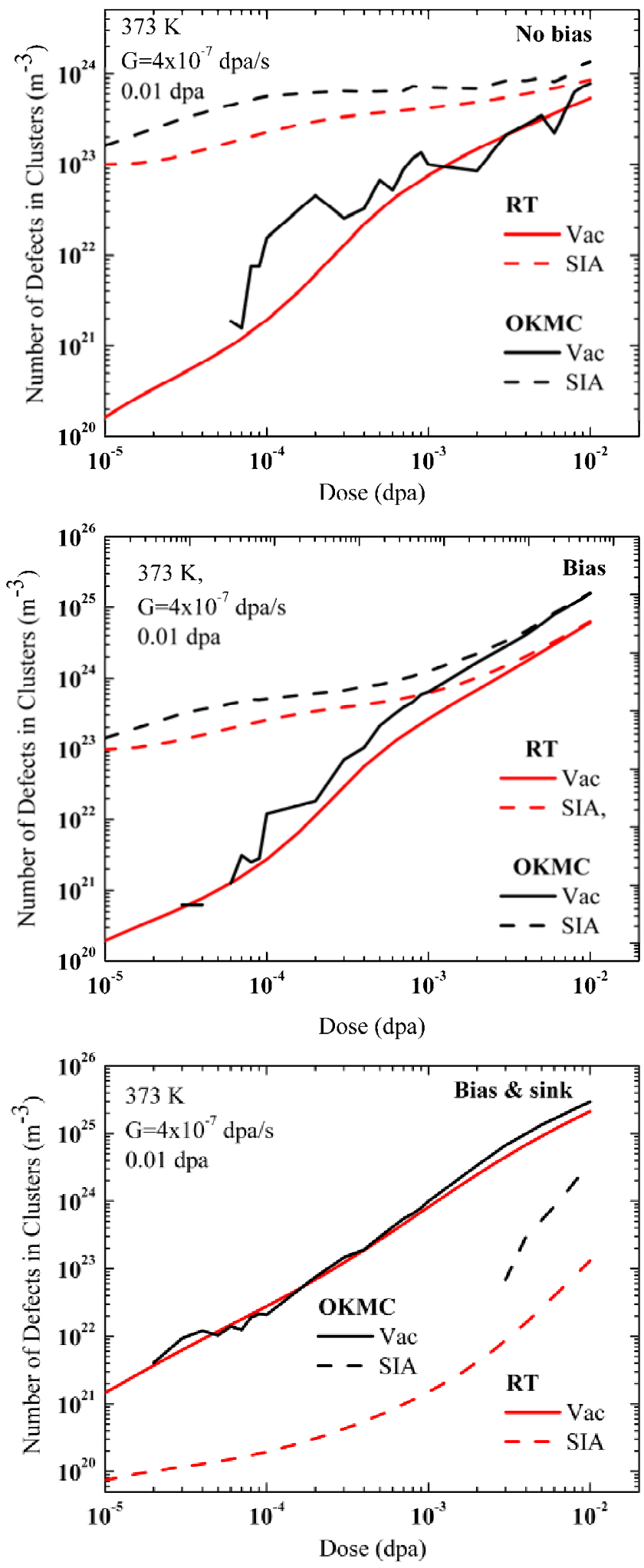

Fig. 7. Number of vacancies and interstitials accumulated in clusters predicted by RT and OKMC for the same three types of sink structures as in Figs. 5 and 6. 

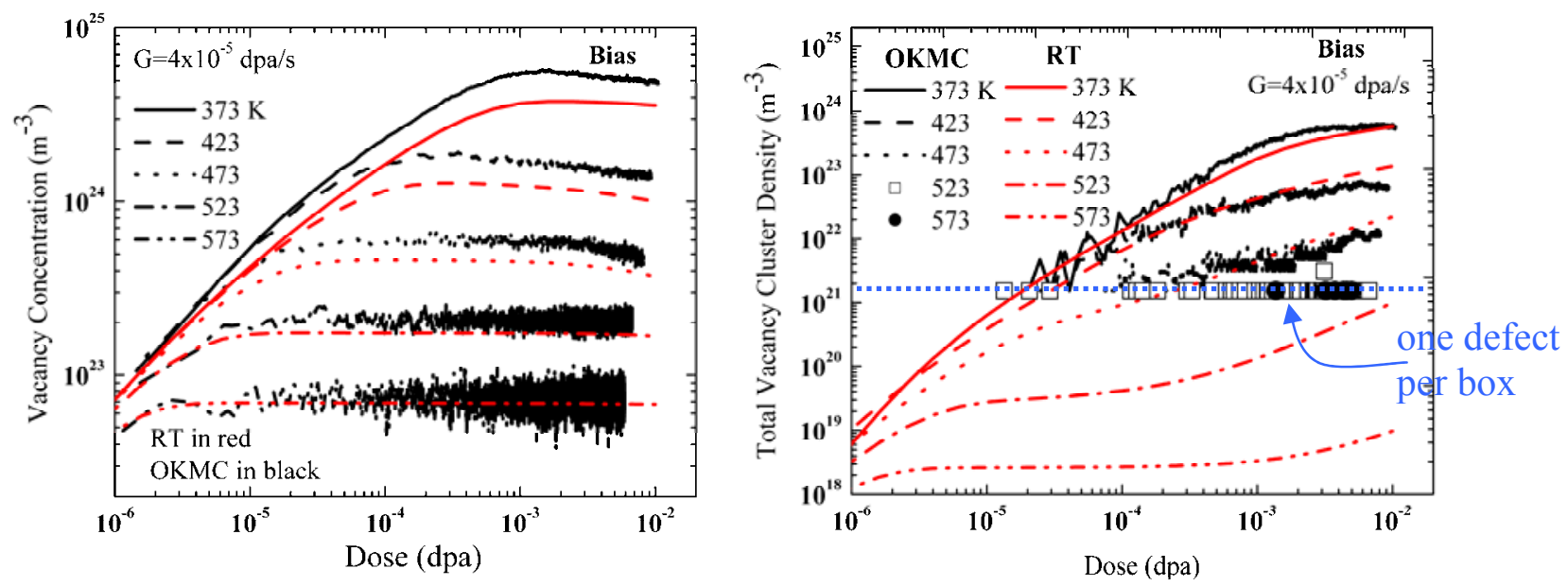

Fig. 8. Influence of irradiation temperature on RT and OKMC predictions of: (a) vacancy concentration and (b) vacancy cluster density.
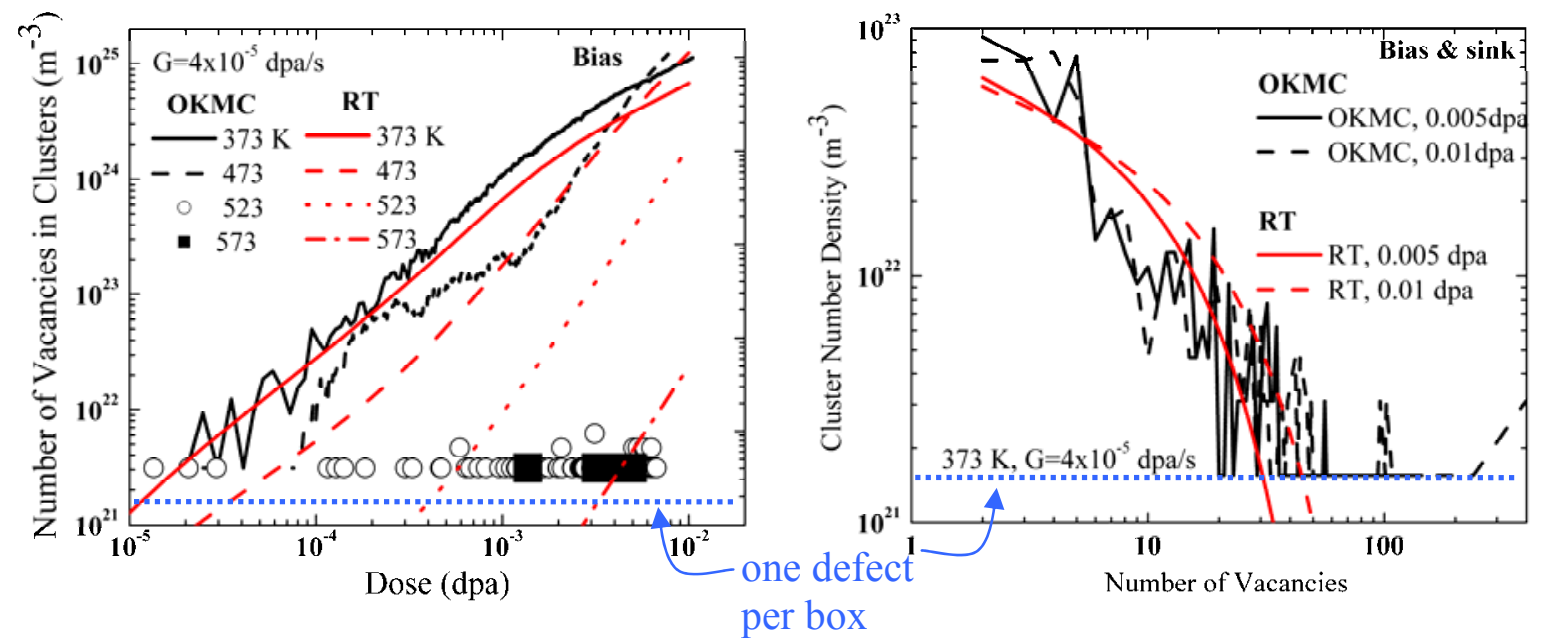

Fig. 9. Influence of irradiation temperature on RT and OKMC predictions of: (a) vacancies accumulated in clusters and (b) vacancy cluster size distribution.

considerable scatter due to the limited number of vacancies and vacancy clusters in the simulation cell. In fact, these results provide a useful measure of the number of objects required for the OKMC model to provide an adequate representation of the RT results. For example, in Fig. 8(a), there is considerable scatter in the value of the vacancy concentration obtained from the $\mathrm{OKMC}$ model at $250^{\circ} \mathrm{C}$. At this temperature, the number of vacancies in the simulation cell is about 100 . At the higher temperature of $300^{\circ} \mathrm{C}$, where there are about 50 vacancies in the simulation cell, the scatter in the vacancy concentration is $+/-50 \%$. The vacancy cluster number 
density falls below one per OKMC cell volume for temperatures greater than $200^{\circ} \mathrm{C}$. The conditions for the size distribution comparison shown in Fig. 9(b) were chosen to have a high cluster density, i.e. $\mathrm{T}=100^{\circ} \mathrm{C}$ and with the biased dislocation sink included. For this case, the RT and OKMC predictions are similar at both 0.005 and $0.01 \mathrm{dpa}$.

Figures 10, 11, and 12 illustrate the impact of atomic displacement rate at a temperature of $100^{\circ} \mathrm{C}$. Results are shown for three displacement rates, $4 \times 10^{-7}, 4 \times 10^{-6}$, and $4 \times 10^{-5} \mathrm{dpa} / \mathrm{s}$. Values of the vacancy concentration obtained from the two models are in good agreement at each damage rate. However, even at these relatively high displacement rates, the interstitial concentration remains well below the density which is equivalent to one object in the OKMC simulation cell. Therefore, it is not possible to obtain an accurate value for the interstitial concentration from the OKMC model. This point is further demonstrated by the point defect cluster densities shown in Fig. 11. Although the two models predict similar values for the void density, substantial fluctuations are observed in the OKMC results as long as the number of voids in the simulation cell is less than a few hundred. Only for the highest displacement rate of $4 \times 10^{-5} \mathrm{dpa} / \mathrm{s}$ is the number of interstitial clusters in the OKMC cell greater than one. Similarly,

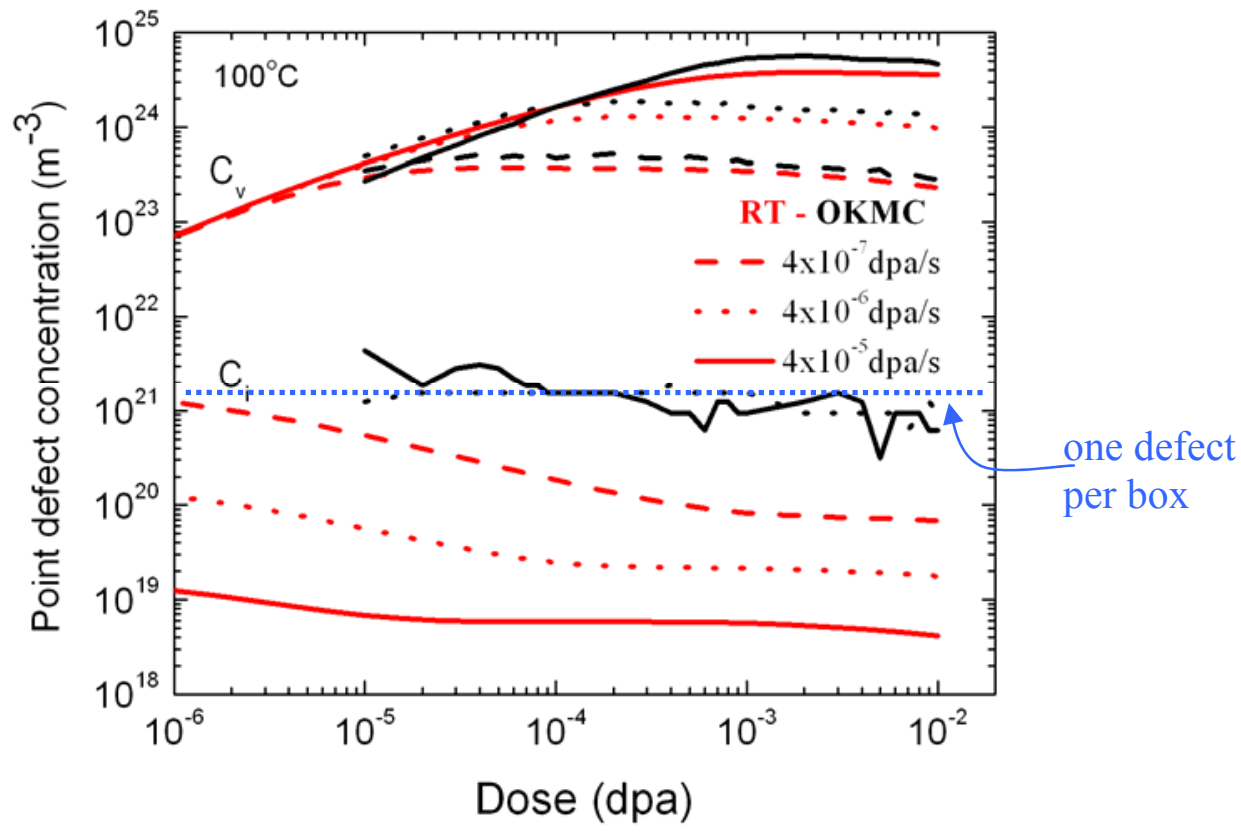

Fig. 10. Influence of atomic displacement rate on point defect concentrations predicted by $\mathrm{RT}$ and $\mathrm{OKMC}$ at $100^{\circ} \mathrm{C}$. 

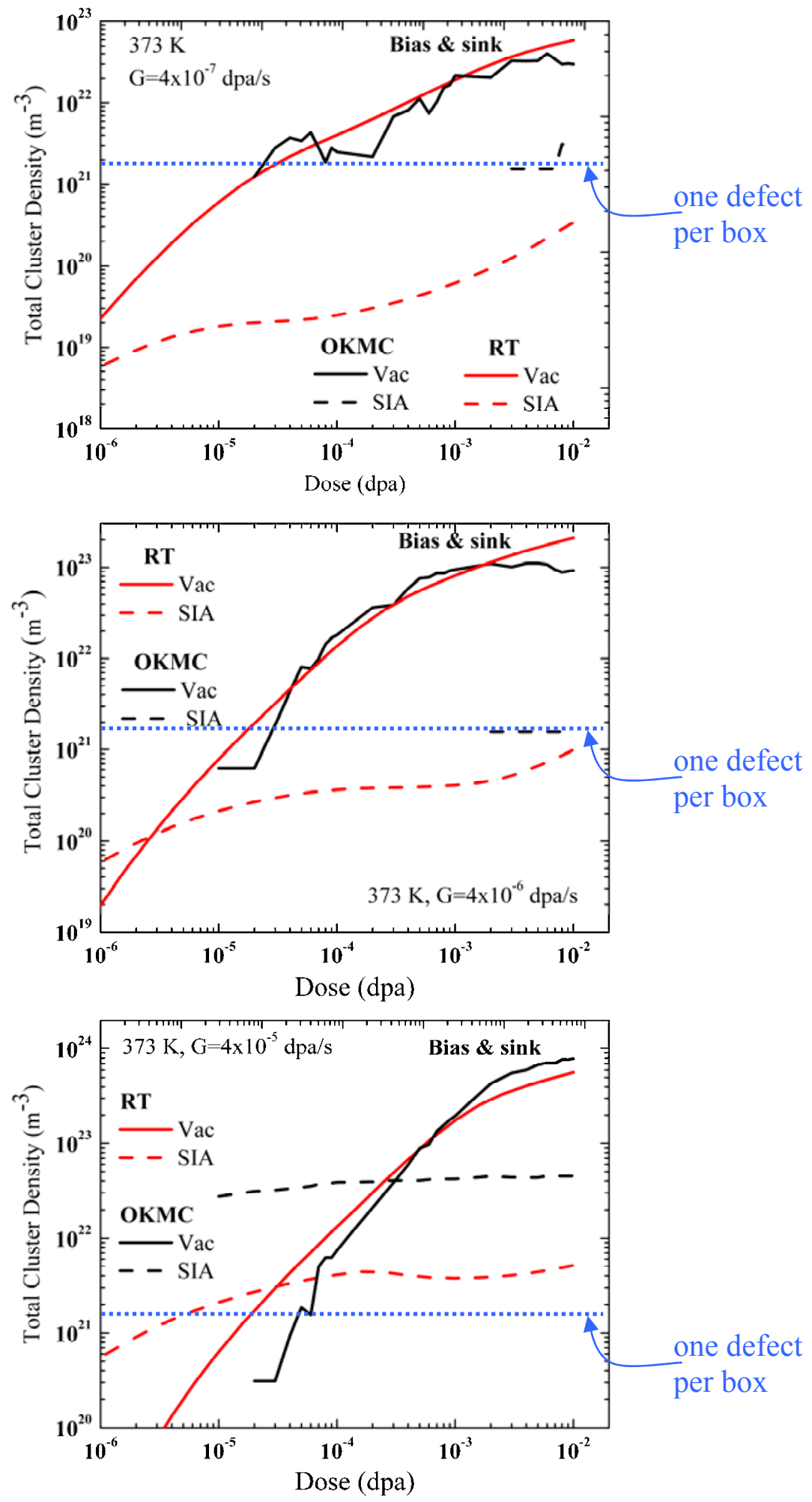

Fig. 11. Influence of atomic displacement rate on RT and OKMC predictions of vacancy and SIA cluster density at $100^{\circ} \mathrm{C}$ for three types of sink structures: (a) only point defect clusters, no biased absorption, (b) same as (a) but interstitial clusters have $20 \%$ bias for SIA, and (c) same as (b) but add fixed dislocation density with $20 \%$ bias for SIA. 

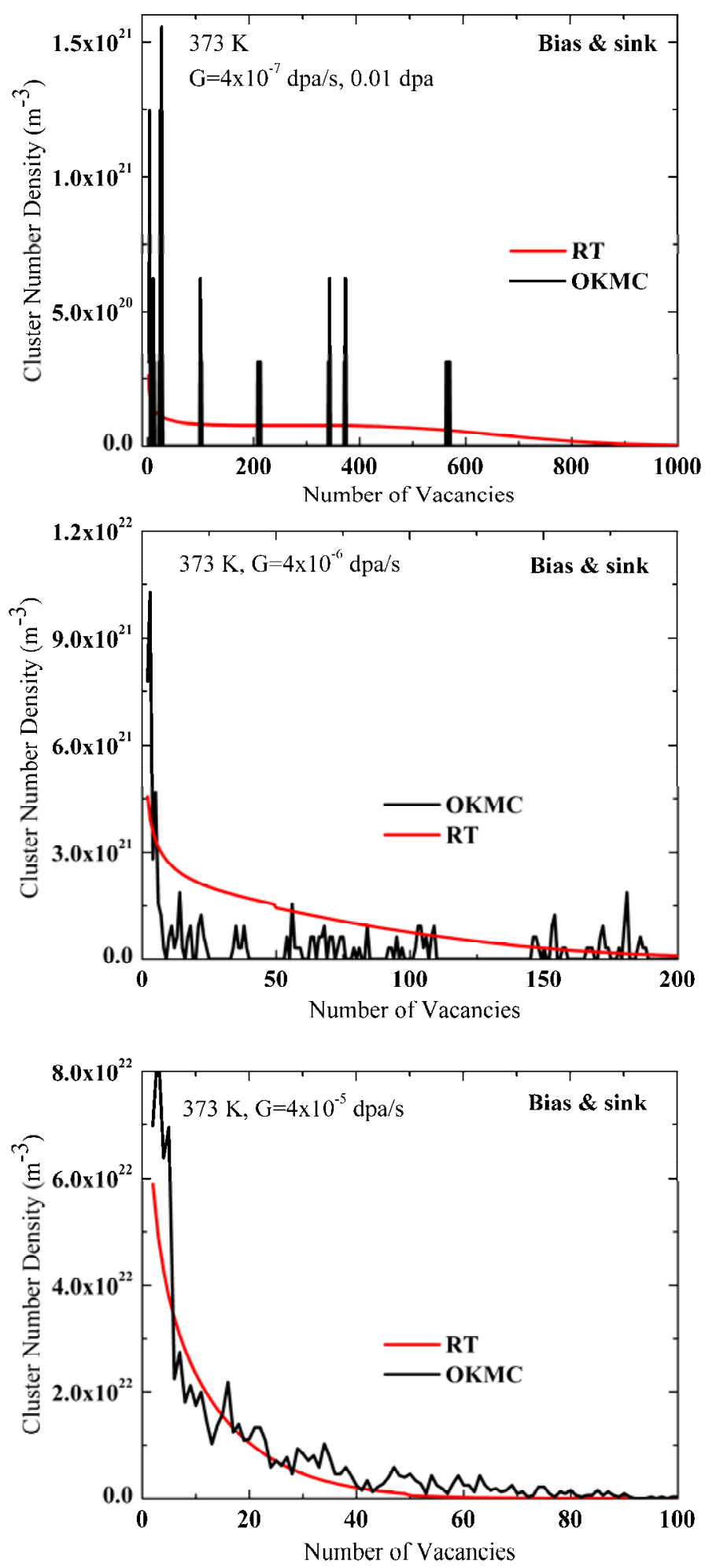

Fig. 12. Influence of atomic displacement rate on RT and OKMC predictions of vacancy cluster size distribution at $100^{\circ} \mathrm{C}$ for the same three types of sink structures as in Fig. 11 . 
Fig. 12 indicates that it is only at this very high displacement rate that the void size distribution function obtained from the OKMC contains enough objects to give a good representation of the SDF obtained from the RT (Fig. 12(c)).

The two sets of involving simulation of cascade damage conditions are summarized in Fig. 13 to 16 . The conditions chosen for this comparison were an NRT displacement rate of $1 \times 10^{-5}$ $\mathrm{dpa} / \mathrm{s}$, cascade efficiency of 0.4 , temperature of $200^{\circ} \mathrm{C}$, and with the most complete sink structure. The cascades used in the OKMC simulations shown in Figs. 13 and 14 are what might be termed realistic cascades, i.e. the spatial correlation of the point defects and small clusters is typical ooc a cascade obtained from molecular dynamics simulations. This spatial correlation was removed in the "cascades" used to obtain the OKMC results shown in Figs. 15 and 16.

Although in-cascade production of point defect clusters increases the cluster density, raising the temperature to $200^{\circ} \mathrm{C}$ reduces the point defect concentrations. As a result, the agreement between the OKMC and RT is relatively poor for the vacancy concentration in Fig. 13(a), the interstitial density is too low to be accurately modeled using OKMC. Values for the point defect cluster densities are in relatively good agreement in Fig. 13(b) since there are a sufficient number of clusters formed. Similarly, the number of vacancies and interstitials
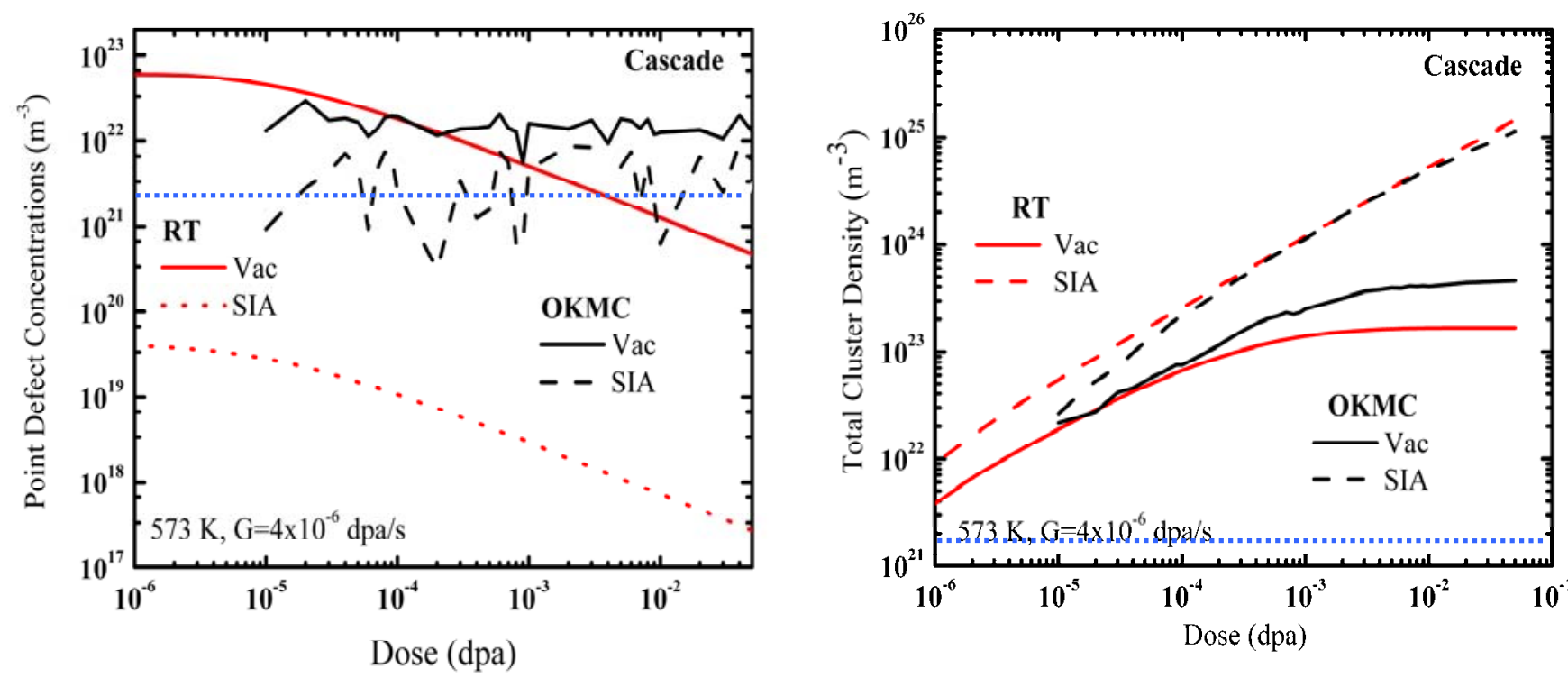

Fig. 13. Dose dependence of RT and OKMC predictions of: (a) point defect concentrations and (b) point defect cluster densities, under cascade damage production with interstitial biased SIA clusters and fixed dislocation sink. Dashed blue lines indicate one defect in OKMC box. 
accumulated in clusters is also in good agreement as seen in Fig. 14(a). The cluster size distributions obtained from the two methods shown in Fig. 14 (b) are in fair agreement at small sizes where a large number of clusters exist. However, the OKMC model can not produce the smooth distribution seen in the RT predictions for large sizes because the density of large clusters is too low.
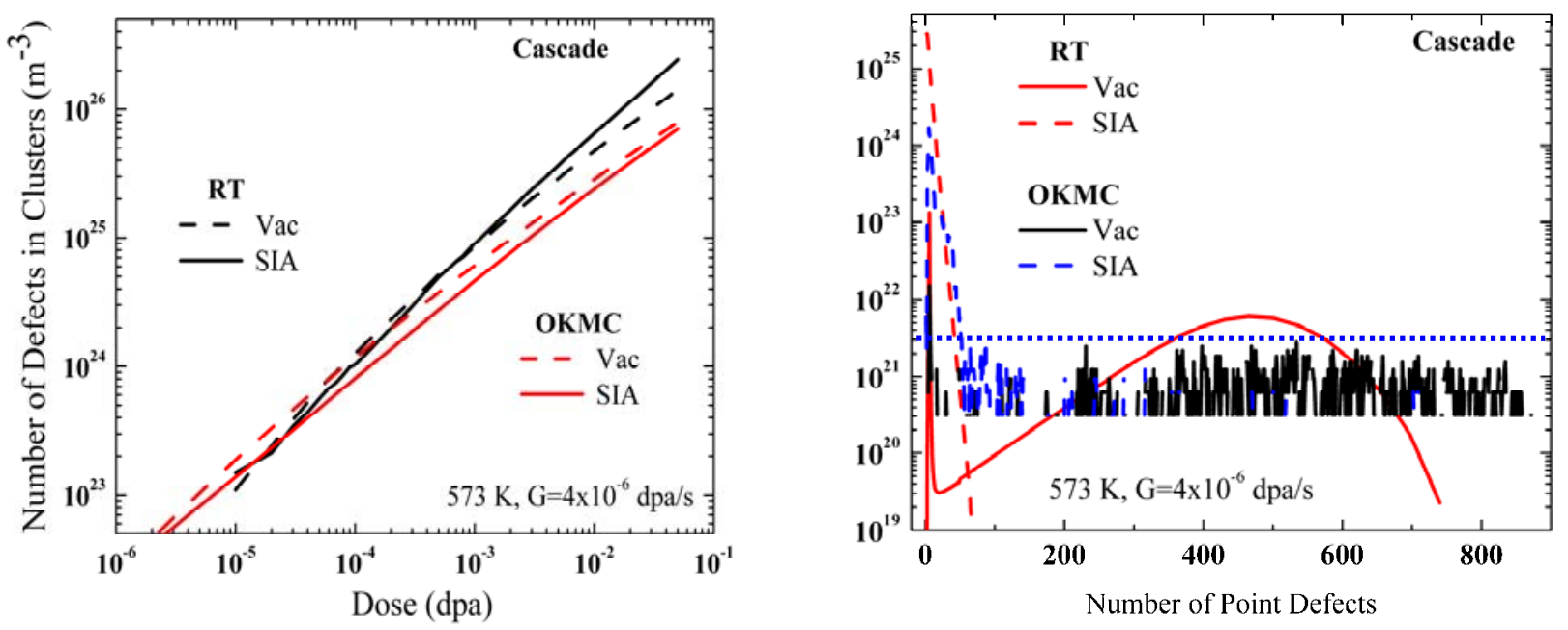

Fig. 14. RT and OKMC predictions of: (a) point defects accumulated in clusters and (b) cluster size distributions, under cascade damage production with interstitial biased SIA clusters and fixed dislocation sink. Dashed blue line in (b) indicates one defect in OKMC box.

Although the simulated cascades used in the OKMC model to produce the results shown in Figs. 15 and 16 are less representative of real atomic displacement cascades, they provide a better simulation of how primary damage production is modeled in the RT. Therefore, it is not surprising that the agreement between the two models is somewhat improved for this case, which can be confirmed by a careful comparison of Fig. 13 with Fig. 15 and Fig. 14 with Fig. 16. Note that the red RT curves are the same in both sets of figures. For example, the OKMC vacancy cluster density in Fig. 15(b) is much closer to the RT result than the corresponding curve in Fig. 13(b), and the agreement for the number of vacancies in clusters is improved between Figs. 14(a) and 14(b). The improvement in the vacancy concentration (Fig. 13(a) vs. Fig. 15(a)) is slight, no doubt due to the limited statistics in the OKMC results. 

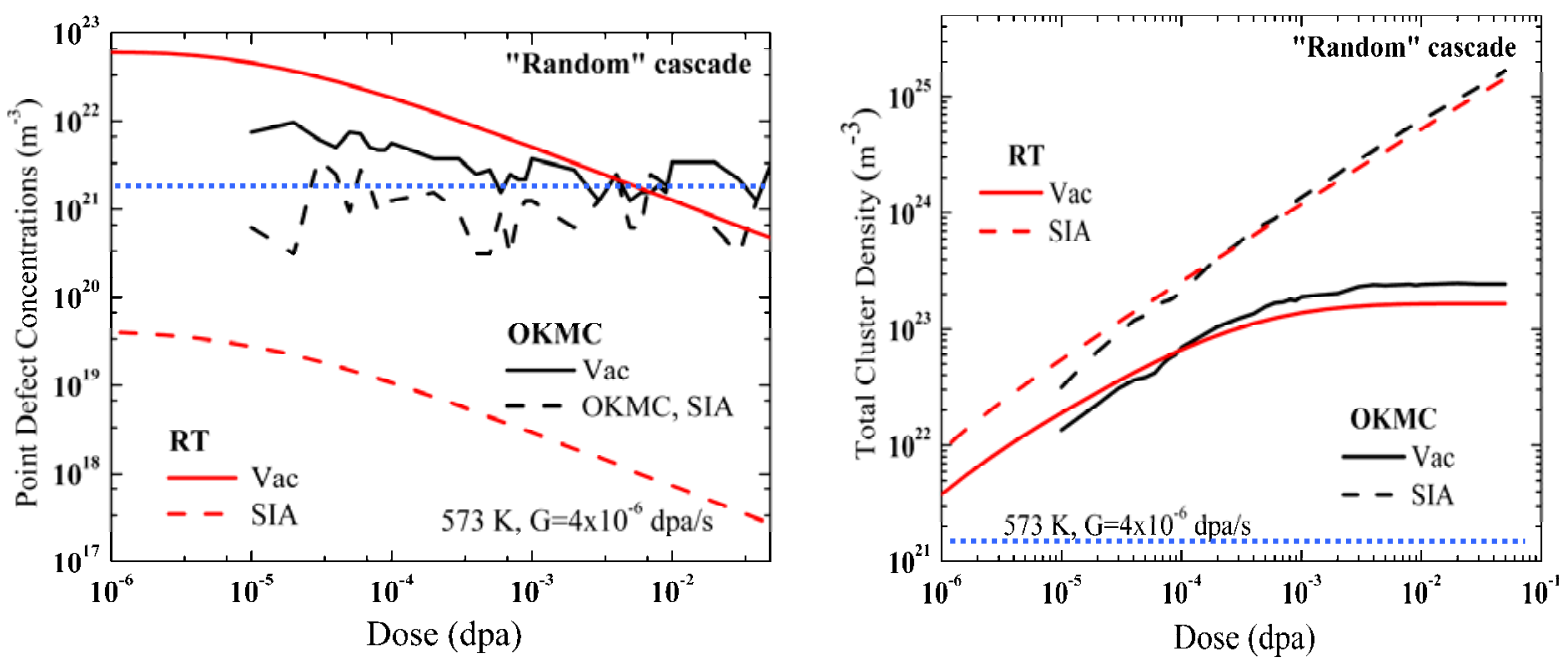

Fig. 15. Dose dependence of RT and OKMC predictions of: (a) point defect concentrations and (b) point defect cluster densities. Cascade damage production in which defects have no spatial correlation, sink structure as in Figs. 13 and 14. Dashed blue lines indicate one defect in OKMC box.
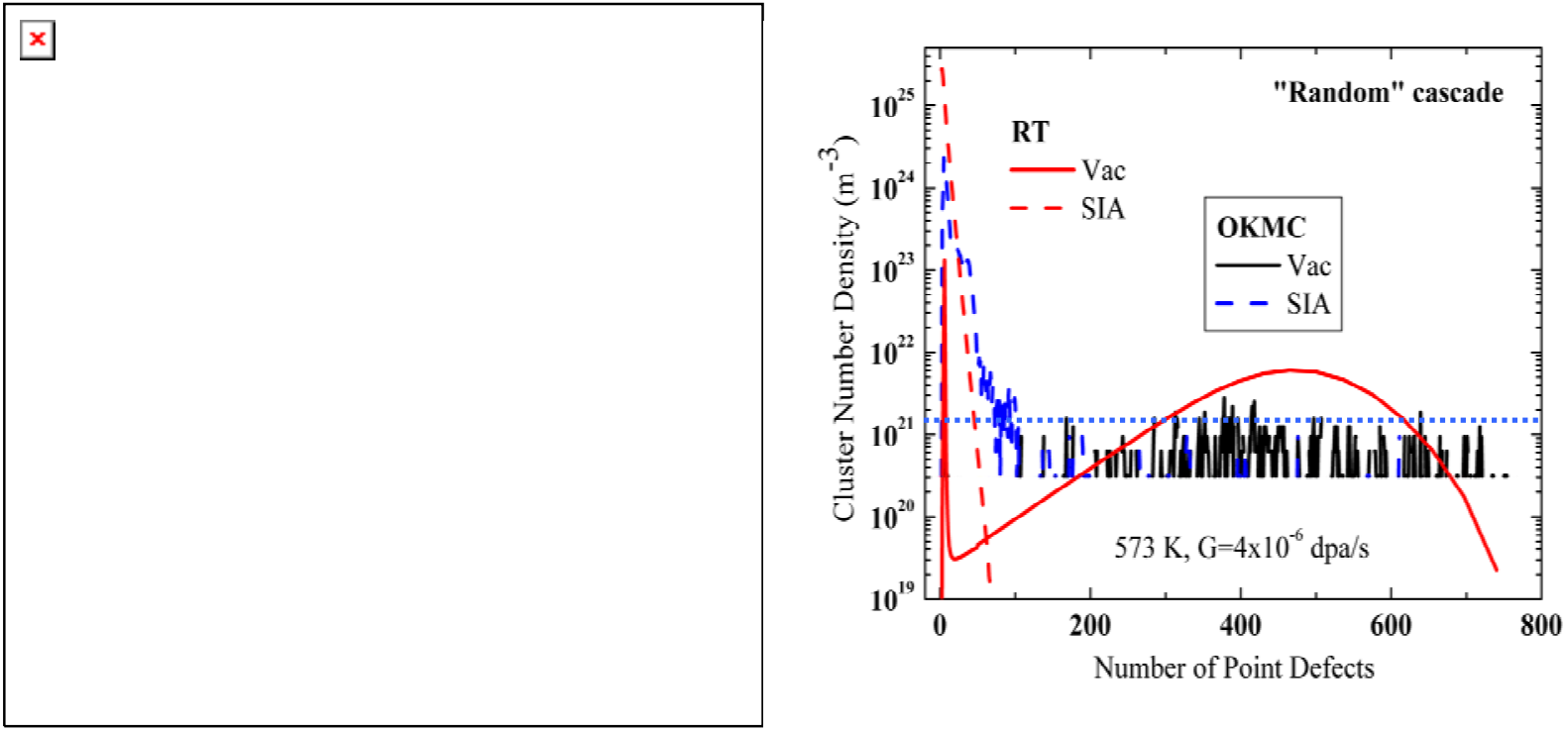

Fig. 16. RT and OKMC predictions of: (a) point defects accumulated in clusters and (b) cluster size distributions. Cascade damage production in which defects have no spatial correlation, sink structure as in Figs. 13 and 14. Dashed blue line in (b) indicates one defect in $\mathrm{OKMC}$ box

\section{Discussion}

The results presented in the preceding section have highlighted both the similarities and differences inherent in the reaction rate theory and object kinetic Monte Carlo methods. They 
confirm that the two methods will give nearly identical results if the problem to be solved is well posed. However, because of the inherent differences in the methods listed in Table 1 and discussed above, specifying the problem in such a way as to obtain the desired equivalence is not a trivial exercise. For example, if small point defect clusters are described in a "standard" way in both approaches, the inherent difference between the OKMC lattice-based reaction rates and the RT continuum sink strengths will lead to systematic difference in the results. The OKMC reaction rates lead to a lower effective sink strength which increases the concentration of monodefects in the matrix. This significance of this difference depends on the details of the problem. It is greater for conditions in which small defect clusters are more dominant, e.g. electron irradiation of a material with a low dislocation density. Lower temperatures and higher damage rates would also increase it's significance.

It may be argued that the OKMC reaction rates are more accurate for the small clusters since a collection of only a few vacancies may be poorly described by the spherical approximation used in the RT sink strengths. Similarly, the ability to account for the spatial correlation of the defects produced by displacement cascades is an advantage of the OKMC relative to the RT. These differences could be accounted for in the RT by using the results specific OKMC simulations to provide deriving a set of correction factors that could be applied in the RT models. For example, information such as that shown in Fig.2 could be used to correct the RT sink strengths for small point defect clusters. Monte Carlo simulations could also be used to determine that degree of additional point defect recombination that occurs due to spatial correlations in cascade debris [19], and a correction could be applied to the RT damage production rate.

The primary limitations of the OKMC model are related to computational issues. Even with modern computers, the maximum simulation cell size and the maximum dose (typically much less than $1 \mathrm{dpa}$ ) that can be simulated are limited. Increasing either of these parameters necessitates a reduction in the other. The limited cell size directly specifies the minimum density of any type of object in the system; no density lower than one object per simulation cell volume can be simulated. Since defect densities are a strong function of irradiation temperature and damage rate, the density limit implies limits on the irradiation conditions that can be simulated. For a given box size, there will be a combination of maximum temperature and minimum dose rate for which the OKMC model can be used.. However, an object density of one per box volume 
is not a sufficient criterion for successful use of the OKMC. Because of the statistical nature of Monte Carlo methods, there should be enough objects of each type to ensure the statistical significance of the results. The results shown in Fig. 8(a) provide the clearest example. Significant fluctuations in the vacancy concentration were observed even when there were as many as 300 vacancies in the simulation cell, partly because there were less than 10 vacancy clusters to act as sinks. Under conditions in which nucleation of extended defects is difficult and proceeds primarily on the basis of fluctuations (high temperature and low damage rate), the OKMC method is unlikely to predict accurate nucleation rates if a statistically significant number of the nucleating species is not present.

In contrast with the OKMC, even very detailed RT models can simulate microstructural evolution for doses up $100 \mathrm{dpa}$ or greater in clock times that are relatively short. Within the context of the effective medium, essentially any defect density can be simulated. Overall, the agreement between the two methods is best for irradiation conditions which produce a high density of defects (lower temperature and higher displacement rate), and for materials that have a relatively high density of fixed sinks such as dislocations. The higher dislocation density reduces the significance of differences associated with the sink strength of small defect clusters..

\section{Summary}

This study has verified the ability of alternate kinetic models based on the reaction rate theory and kinetic Monte Carlo methods to obtain comparable results in well-posed simulations that are directly relevant to modeling radiation-induced microstructural evolution. It has also help define irradiation regimes in which it may be inappropriate to use the OKMC methods. In particular, current computational limitations on the OKMC simulation cell size imply that it may be difficult to use the OKMC at displacement rates and temperatures relevant to many reactor components. Expected advances in computing will expand the range of use for OKMC models, but this progress may occur slowly since orders of magnitude in defect density are required. However, the OKMC does a better job of accounting for spatial correlations that influence point defect reaction rates with small defect clusters and that modify the primary damage source term under cascade damage conditions. As such, one immediate application of OKMC simulations is to improve the parameterization of the rate theory models.

\section{References}


1. S. I. Golubov, R. E. Stoller, C. S. Becquart, and C. Domain, "Preliminary Comparison of Reaction Rate Theory and Object Kinetic Monte Carlo Simulations of Defect Cluster Dynamics under Irradiation," ORNL/GEN4/LTR-06-030, Oak Ridge National Laboratory, 2006.

2. L. K. Mansur, "Mechanisms and Kinetics of Radiation Effects in Metals and Alloys," Kinetics of Nonhomogeneous Processes, Wiley-Interscience, New York, 1987, pp. 377-463.

3. M. Kiritani, J. Phys. Soc. Japan 35 (1973) 95.

4. J. L. Katz and H. Wiedersich, J. Chem. Phys. 55 (1973) 1414.

5. N. M. Ghoniem and S. Sharafat, J. Nucl. Mater. 92 (1980) 121.

6. S. I. Golubov, A. M. Ovcharenko, A. V. Barashev, and B. N. Singh, Philos. Mag. A 81 (2001) 643.

7. R. E. Stoller and G. R. Odette, "A Composite Model of Microstructural Evolution in Austenitic Stainless Steel Under Fast Neutron Irradiation,” Radiation-Induced Changes in Microstructure, ASTM STP 955, F. A. Garner, N. H. Packan, and A. S. Kumar, Eds., ASTM International, West Conshohocken, PA, 1987, pp. 371-392.

8. M. P. Surh, J. B. Sturgeon, W.G. Wolfer, J. Nucl. Mater. 325 (2004) 44.

9. N. Metropolis, A.W. Rosenbluth, M.N. Rosenbluth, A.H. Teller and E. Teller, J. Chem. Phys. 21 (1953) 1087.

10. W. A. Coghlan and L. K. Mansur, J. Nucl. Mater. 108\&109 (1982) 246.

11. S. I. Golubov, S. I., Phys. Metals Metallogr. (USSR) 52 (1981) 86.

12. C. Domain, C. S. Becquart, and L. Malerba, J. Nucl. Mater. 335 (2004) 121.

13. W. M. Young and E. W. Elcock, Proc. Phys. Soc. 89 (1966) 735.

14. B. Bortz, M. H. Kalos, J. L. Lebowitz, J. Comp. Phys. 17 (1975) 10.

15. A. D. Brailsford and R. Bullough, "The Theory of Sink Strengths," Philos. Trans. of the Royal Society of London, Series A, Vol. 302 (1981), pp. 87-137.

16. R. E. Stoller and A. F. Calder, J. Nucl. Mater. 283-287 (2000) 746-752.

17. H. L. Heinisch, B. N. Singh, and S. I. Golubov, J. Computer-Aided Mat. Design, 6 (1999) 277.

18. S. I. Golubov, B. N. Singh, and H. Trinkaus, Phil. Mag. A 81 (2001) 2533.

19. B. D. Wirth, G. R. Odette, and R. E. Stoller, "Recent Progress Toward an Integrated Multiscale-Multiphysics Model of Reactor Pressure Vessel Embrittlement," Advances in Materials Theory and Modeling - Bridging Over Multiple-Length and Time Scales, MRS Vol. 677, V. Bulatov, F. Cleri, L. Colombo, L. Lewis, and N. Mousseau, Eds. Materials Research Society, Pittsburgh, PA, 2001, pp. 5.2.1-5.2.6. 


\section{List of Tables}

Table 1: Comparison of reaction rate theory and object kinetic Monte Carlo methods

Table 2. Material and irradiation parameters used in calculations 


\section{Figure Captions}

Fig. 1. Void size distribution function calculated for the case of $E_{2 v}=0.3: F(x)$ in phase space $x$ ( $x=$ number of vacancies), and $F(d)$ in phase space $d$ ( $d=$ void diameter).

Fig. 2. Comparison of vacancy cluster sink strength obtained in OKMC simulations (discrete points) and the analytical expression applied in the RT (lines). The dashed line is the lowest order sink strength, which is valid for small sink volume fractions, and the solid line includes the first order sink strength correction factor [15].

Fig 3. Vacancy concentration for the case of only Frenkel pair production and three sink variants (see text).

Fig. 4. Dose dependence of vacancy and interstitial cluster density predicted by RT and OKMC for three types of sink structures: (a) only point defect clusters, no biased absorption, (b) same as (a) but interstitial clusters have $20 \%$ bias for SIA, and (c) same as (b) but add fixed dislocation density with $20 \%$ bias for SIA.

Fig. 5. Vacancy cluster size distributions predicted by RT and OKMC for three types of sink structures: (a) only point defect clusters, no biased absorption, (b) same as (a) but interstitial clusters have $20 \%$ bias for SIA, and (c) same as (b) but add fixed dislocation density with $20 \%$ bias for SIA.

Fig. 6. Interstitial cluster size distributions predicted by RT and OKMC for the same three types of sink structures as in Fig. 5.

Fig. 7. Number of vacancies and interstitials accumulated in clusters predicted by RT and OKMC for the same three types of sink structures as in Figs. 5 and 6.

Fig. 8. Influence of irradiation temperature on RT and OKMC predictions of: (a) vacancy concentration and (b) vacancy cluster density.

Fig. 9. Influence of irradiation temperature on RT and OKMC predictions of: (a) vacancies accumulated in clusters and (b) vacancy cluster size distribution.

Fig. 10. Influence of atomic displacement rate on point defect concentrations predicted by RT and $\mathrm{OKMC}$ at $100^{\circ} \mathrm{C}$.

Fig. 11. Influence of atomic displacement rate on RT and OKMC predictions of vacancy and SIA cluster density at $100^{\circ} \mathrm{C}$ for three types of sink structures: (a) only point defect clusters, no biased absorption, (b) same as (a) but interstitial clusters have $20 \%$ bias for SIA, and (c) same as (b) but add fixed dislocation density with $20 \%$ bias for SIA.

Fig. 12. Influence of atomic displacement rate on RT and OKMC predictions of vacancy cluster size distribution at $100^{\circ} \mathrm{C}$ for the same three types of sink structures as in Fig. 11.

Fig. 13. Dose dependence of RT and OKMC predictions of: (a) point defect concentrations and (b) point defect cluster densities, under cascade damage production with interstitial biased SIA clusters and fixed dislocation sink.

Fig. 14. RT and OKMC predictions of: (a) point defects accumulated in clusters and (b) cluster size distributions, under cascade damage production with interstitial biased SIA clusters and fixed dislocation sink.

Fig. 15. Dose dependence of RT and OKMC predictions of: (a) point defect concentrations and 
(b) point defect cluster densities. Cascade damage production in which defects have no spatial correlation, sink structure as in Figs. 13 and 14.

Fig. 16. RT and OKMC predictions of: (a) point defects accumulated in clusters and (b) cluster size distributions. Cascade damage production in which defects have no spatial correlation, sink structure as in Figs. 13 and 14. 